\title{
A Carleson-type estimate in Lipschitz type domains for non-negative solutions to Kolmogorov operators
}

\author{
Chiara Cinti, Kaj Nyström and Sergio Polidoro
}

\begin{abstract}
We prove a Carleson type estimate, in Lipschitz type domains, for non-negative solutions to a class of second order degenerate differential operators of Kolmogorov type of the form

$$
\mathscr{L}=\sum_{i, j=1}^{m} a_{i, j}(z) \partial_{x_{i} x_{j}}+\sum_{i=1}^{m} a_{i}(z) \partial_{x_{i}}+\sum_{i, j=1}^{N} b_{i, j} x_{i} \partial_{x_{j}}-\partial_{t},
$$

where $z=(x, t) \in \mathbb{R}^{N+1}, 1 \leq m \leq N$. Our estimate is scale-invariant and generalizes previous results valid for second order uniformly parabolic equations to the class of operators considered.
\end{abstract}

Mathematics Subject Classification (2010): 35K65 (primary); 35K70, 35H20, 35R03 (secondary).

\section{Introduction}

In this paper we prove a Carleson type estimate for non-negative solutions to a general class of hypoelliptic ultraparabolic operators. Specifically, we consider Kolmogorov operators of the form

$$
\mathscr{L}=\sum_{i, j=1}^{m} a_{i, j}(z) \partial_{x_{i} x_{j}}+\sum_{i=1}^{m} a_{i}(z) \partial_{x_{i}}+\sum_{i, j=1}^{N} b_{i, j} x_{i} \partial_{x_{j}}-\partial_{t},
$$

where $z=(x, t) \in \mathbb{R}^{N} \times \mathbb{R}, 1 \leq m \leq N$, the coefficients $a_{i, j}$ and $a_{i}$ are bounded continuous functions and $B=\left(b_{i, j}\right)_{i, j=1, \ldots, N}$ is a matrix of real constants. The main motivation for our research is our long-term goal to establish a regularity theory for the free boundaries occurring in the obstacle problem

$$
\begin{cases}\max \{\mathscr{L} u, \varphi-u\}=0, & \text { in } \left.\mathbb{R}^{N} \times\right] 0, T[ \\ u(x, 0)=\varphi(x, 0), & \text { for any } x \in \mathbb{R}^{N}\end{cases}
$$

Received March 14, 2011; accepted August 22, 2011. 
considered by Di Francesco, Pascucci and Polidoro in [10]. In [21] and [32] Frentz, Nyström, Pascucci and Polidoro proved optimal regularity properties of the solution to the obstacle problem, for smooth as well as non-smooth obstacles. Up to date, the only known results about boundary regularity for non-negative solutions to Kolmogorov operators have been proved by the authors in [6], see also [7].

Our long-term goal is to establish a boundary regularity theory for Kolmogorov operators. Estimates concerning boundary behaviour are of obvious independent interest from the theoretical point of view. Furthermore, the regularity properties of the Kolmogorov equations on $\mathbb{R}^{N+1}$ depend strongly on a geometric Lie group structure. The extension of the methods used in the Euclidean setting to the Lie group geometry related to Kolmogorov equations is far from trivial. For results concerning the boundary behaviour of non-negative solutions and obstacle problems in different, but still subelliptic settings, we refer to the works by Frentz, Garofalo, Götmark, Munive and Nyström [20], Capogna, Garofalo and Nhieu [4,5], Franchi and Ferrari [18, 19], Danielli, Garofalo and Salsa [9], Danielli, Garofalo and Petrosyan [8].

We next list our structural assumptions on the operator $\mathscr{L}$ defined in (1.1).

[H.1] The matrix $A_{0}(z)=\left(a_{i, j}(z)\right)_{i, j=1, \ldots, m}$ is symmetric and uniformly positive definite in $\mathbb{R}^{m}$ : there exists a positive constant $\lambda$ such that

$$
\lambda^{-1}|\xi|^{2} \leq \sum_{i, j=1}^{m} a_{i, j}(z) \xi_{i} \xi_{j} \leq \lambda|\xi|^{2}, \quad \forall \xi \in \mathbb{R}^{m}, z \in \mathbb{R}^{N+1}
$$

The matrix $B=\left(b_{i, j}\right)_{i, j=1, \ldots, N}$ has real constant entries.

[H.2] The constant coefficients operator

$$
\mathscr{K}=\sum_{i, j=1}^{m} a_{i, j} \partial_{x_{i} x_{j}}+\sum_{i, j=1}^{N} b_{i, j} x_{i} \partial_{x_{j}}-\partial_{t}
$$

is hypoelliptic, i.e. every distributional solution of $\mathscr{K} u=f$ is a smooth classical solution, whenever $f$ is smooth. Here $A_{0}=\left(a_{i, j}\right)_{i, j=1, \ldots, m}$ is any constant, symmetric and strictly positive matrix.

[H.3] The coefficients $a_{i, j}(z)$ and $a_{i}(z)$ are bounded functions belonging to the Hölder space $\left.\left.C_{K}^{0, \alpha}\left(\mathbb{R}^{N+1}\right), \alpha \in\right] 0,1\right]$, defined in (2.6) below.

Note that we can choose $A_{0}$ in [H.2] as the $m \times m$ identity matrix. In our setting, $\mathscr{K}$ plays the same role as the heat operator does in the framework of uniformly parabolic pdes. We also note that the operator $\mathscr{K}$ can be written as

$$
\mathscr{K}=\sum_{i=1}^{m} X_{i}^{2}+Y,
$$

where

$$
X_{i}=\sum_{j=1}^{m} \bar{a}_{i, j} \partial_{x_{j}}, \quad i=1, \ldots, m, \quad Y=\langle x, B \nabla\rangle-\partial_{t},
$$


and where $\bar{a}_{i, j}$ 's are the entries of the unique positive matrix $\bar{A}_{0}$ such that $A_{0}=\bar{A}_{0}^{2}$. Recall that the hypothesis [H.2] is equivalent to the Hörmander condition [24],

$$
\operatorname{rank} \operatorname{Lie}\left(X_{1}, \ldots, X_{m}, Y\right)(z)=N+1, \quad \forall z \in \mathbb{R}^{N+1} .
$$

It is well-known that the natural framework to study operators satisfying a Hörmander condition is the analysis on Lie groups. The relevant Lie group related to the operator $\mathscr{K}$ in (1.2) is defined using the group law

$$
(x, t) \circ(\xi, \tau)=\left(\xi+\exp \left(-\tau B^{T}\right) x, t+\tau\right), \quad(x, t),(\xi, \tau) \in \mathbb{R}^{N+1} .
$$

In particular, the vector fields $X_{1}, \ldots, X_{m}$ and $Y$ are left-invariant, with respect to the group law (1.5), in the sense that

$$
X_{j}(u(\zeta \circ \cdot))=\left(X_{j} u\right)(\zeta \circ \cdot), \quad j=1, \ldots, m, \quad Y(u(\zeta \circ \cdot))=(Y u)(\zeta \circ \cdot),
$$

for every $\zeta \in \mathbb{R}^{N+1}$ (hence $\left.\mathscr{K}(u(\zeta \circ \cdot))=(\mathscr{K} u)(\zeta \circ \cdot)\right)$. It is also known that [H.2] is equivalent to the following structural assumption on $B$ [28]: there exists a basis for $\mathbb{R}^{N}$ such that the matrix $B$ has the form

$$
\left(\begin{array}{ccccc}
* & B_{1} & 0 & \cdots & 0 \\
* & * & B_{2} & \cdots & 0 \\
\vdots & \vdots & \vdots & \ddots & \vdots \\
* & * & * & \cdots & B_{\kappa} \\
* & * & * & \cdots & *
\end{array}\right)
$$

where $B_{j}$ is a $m_{j-1} \times m_{j}$ matrix of rank $m_{j}$ for $j \in\{1, \ldots, \kappa\}, 1 \leq m_{\kappa} \leq \ldots \leq$ $m_{1} \leq m_{0}=m$ and $m+m_{1}+\ldots+m_{\kappa}=N$, while $*$ represents arbitrary matrices with constant entries. Based on (1.7), we introduce the family of dilations $\left(\delta_{r}\right)_{r>0}$ on $\mathbb{R}^{N+1}$ defined by

$$
\delta_{r}=\left(D_{r}, r^{2}\right)=\operatorname{diag}\left(r I_{m}, r^{3} I_{m_{1}}, \ldots, r^{2 \kappa+1} I_{m_{\kappa}}, r^{2}\right),
$$

where $I_{k}, k \in \mathbb{N}$, is the $k$-dimensional unit matrix. To simplify our presentation, we will also assume the following technical condition.

[H.4] The operator $\mathscr{K}$ in (1.2) is $\delta_{r}$-homogeneous of degree two, i.e.

$$
\mathscr{K} \circ \delta_{r}=r^{2}\left(\delta_{r} \circ \mathscr{K}\right), \quad \forall r>0 .
$$

We explicitly remark that [H.4] is satisfied if (and only if) all the blocks denoted by $*$ in (1.7) are null (see [28]). Moreover we set

$$
\mathbf{q}=m+3 m_{1}+\ldots+(2 \kappa+1) m_{\kappa},
$$

and we say that $\mathbf{q}+2$ is the homogeneous dimension of $\mathbb{R}^{N+1}$ with respect to the dilations group $\left(\delta_{r}\right)_{r>0}$. 
Consider the boundary value problem

$$
\begin{cases}\mathscr{L} u=0 & \text { in } \Omega, \\ u=\varphi & \text { in } \partial \Omega,\end{cases}
$$

where $\Omega$ is any open subset of $\mathbb{R}^{N+1}$ and $\varphi \in C(\partial \Omega)$. Using the Perron-WienerBrelot method, the existence of a solution to this problem can be established. However, it is well known that the boundary value $\varphi$ is not necessarily attained at every point of $\partial \Omega$. In the sequel, $u_{\varphi}$ will denote the solution to (1.9), and we set

$$
\partial_{K} \Omega=\left\{z \in \partial \Omega \mid \lim _{w \rightarrow z} u_{\varphi}(w)=\varphi(z) \text { for any } \varphi \in C(\partial \Omega)\right\} .
$$

We refer to $\partial_{K} \Omega$ as the regular boundary of $\Omega$ with respect to the operator $\mathscr{L}$. We recall that Manfredini in [30, Proposition 6.1] gives sufficient conditions for the regularity of the boundary points. Recall that a vector $v \in \mathbb{R}^{N+1}$ is an outer normal to $\Omega$ at $z \in \partial \Omega$ if there exists a positive $r$ such that $B(z+r v, r) \cap \Omega=\emptyset$. Here $B(z+r v, r)$ denotes the Euclidean ball in $\mathbb{R}^{N+1}$ with center at $z+r v$ and radius $r$. Then, in consistency with Fichera's classification, sufficient conditions for the regularity are expressed in geometric terms, and read as follows. If $z \in \partial \Omega$ and $v=\left(v_{1}, \ldots, v_{N+1}\right)$ is an outer normal to $\Omega$ at $z$, then the following holds.

(a) If $\left(v_{1}, \ldots, v_{m}\right) \neq 0$, then $z \in \partial_{K} \Omega$,

(b) if $\left(v_{1}, \ldots, v_{m}\right)=0$ and $\langle Y(z), v\rangle>0$, then $z \in \partial_{K} \Omega$,

(c) if $\left(v_{1}, \ldots, v_{m}\right)=0$ and $\langle Y(z), v\rangle<0$, then $z \notin \partial_{K} \Omega$,

where $Y$ is the vector field defined in (1.3). As an example, we consider the simplest Kolmogorov operator $\partial_{x_{1}}^{2}+x_{1} \partial x_{2}-\partial_{t}$ in the set $\left.\Omega=\right]-1,1[\times]-1,1[\times]-1,0[\subset$ $\mathbb{R}^{3}$. Then $Y(x, t)=\left(0, x_{1},-1\right)$, and it is easy to see that

(a) is satisfied by all the points of the set $\{-1,1\} \times]-1,1[\times]-1,0[$,

(b) is satisfied by all the points of the set $]-1,1[\times]-1,1[\times\{-1\} \cup$

$$
\cup] 0,1[\times\{1\} \times]-1,0[\cup]-1,0[\times\{-1\} \times]-1,0[\text {, }
$$

(c) is satisfied by all the points of the set $]-1,1[\times]-1,1[\times\{0\} \cup$

$$
\cup] 0,1[\times\{-1\} \times]-1,0[\cup]-1,0[\times\{1\} \times]-1,0[\text {. }
$$

Condition (a) can be equivalently expressed in terms of the vector fields $X_{j}$ 's as follows: $\left\langle X_{j}(z), v\right\rangle \neq 0$ for some $j=1, \ldots, m$. If this condition holds, in the literature $z$ is often referred to as a non characteristic point for the operator $\mathscr{L}$. A more refined sufficient condition for the regularity of the boundary points of $\partial \Omega$ is given in [30, Theorem 6.3] in terms of an exterior cone condition and that condition will be used here (see Definition 4.1 below).

In [6, Theorem 1.2] we proved a Carleson type estimate for non-negative solutions $u$ to $\mathscr{L} u=0$ assuming that $u$ vanishes continuously on some open subset $\Sigma$ 
of $\partial \Omega$. Furthermore, in [6, Proposition 1.4] we gave an application of [6, Theorem 1.2], assuming that $\Sigma$ is a $N$-dimensional $C^{1}$-manifold satisfying either condition (a) or condition (b) in (1.11). The purpose of this paper is to improve on these results by relaxing the regularity assumptions on $\Sigma$. Specifically, in this paper we assume that $\Sigma$ is locally a $\operatorname{Lip}_{K}$ surface, where $\operatorname{Lip}_{K}$ is a notion of Lipschitz regularity suitably defined in terms of the dilations (1.8) and introduced in the bulk of the paper. We also improve on [6, Theorem 1.2] since our main result, which is stated below, is scale invariant in the sense that $(\tilde{x}, \widetilde{t}), C$ and $c$ do not depend on $\left.r \in] 0, r_{0}\right]$. In the following we refer to Definition 2.1 for the meaning of $\operatorname{Lip}_{K}$ surfaces, and to (2.9), (2.10) for the definition of the cube $Q_{M, r}\left(x_{0}, t_{0}\right)$.

The main result proved in this paper reads as follows.

Theorem 1.1. Let $\Omega$ be an open subset of $\mathbb{R}^{N+1}$, and let $\Sigma \subset \partial \Omega$ be a compact $\operatorname{Lip}_{K}$ surface with constants $M$ and $r_{0}$. Then there exist two positive constants $C, c$, depending only on $\mathscr{L}$ and $M$, such that

$$
\sup _{Q_{M, c r}\left(x_{0}, t_{0}\right) \cap \Omega} u(x, t) \leq C u\left(A_{r}^{+}\left(x_{0}, t_{0}\right)\right), \quad A_{r}^{+}\left(x_{0}, t_{0}\right)=\left(x_{0}, t_{0}\right) \circ \delta_{r}(\tilde{x}, \tilde{t}),
$$

for any $\left(x_{0}, t_{0}\right) \in \Sigma$, for every non-negative solution $u$ to $\mathscr{L} u=0$ in $\Omega$ vanishing continuously on $Q_{M, r}\left(x_{0}, t_{0}\right) \cap \partial \Omega$, and for every $\left.\left.r \in\right] 0, r_{0}\right]$. Here $(\widetilde{x}, \widetilde{t}) \in \mathbb{R}^{N+1}$ is such that

$$
\|(\tilde{x}, \tilde{t})\|_{K} \leq C, \quad d_{K}\left(A_{r}^{+}\left(x_{0}, t_{0}\right), \partial \Omega\right) \geq c r,
$$

for every $r \in] 0, r_{0}[$.

To put Theorem 1.1 in the context of the existing literature devoted to boundary estimates for second order elliptic and parabolic operators, we here briefly discuss previous results valid for uniformly parabolic operators of the form

$$
L=\sum_{i, j=1}^{N} a_{i j}(x, t) \partial_{x_{i} x_{j}}-\partial_{t}, \quad(x, t) \in \mathbb{R}^{N+1},
$$

where the matrix $\left(a_{i, j}(x, t)\right)$ satisfies [H.1] with $m=N$. The study of the boundary behavior of non-negative solutions to non-divergence form uniformly parabolic equations $L u=0$, as well as the associated $L$-parabolic measure, has a long history. We quote the papers by Fabes and Kenig [14], Fabes and Stroock [17], Garofalo [22], Krylov and Safonov [27], leading up to the results of Fabes, Safonov and Yuan in [16] and [33]. The corresponding developments for second order parabolic operators in divergence form are treated by Fabes, Garofalo and Salsa [13], Fabes and Safonov [15], Nyström [31]. We refer to Bauman [1], Caffarelli, Fabes, Mortola and Salsa [2], Fabes, Garofalo, Marin-Malave and Salsa [12], and Jerison and Kenig [25] for both divergence and non-divergence form elliptic operators. Finally, we also note that second order elliptic and parabolic operators in divergence form with singular lower order terms have been studied by Kenig and Pipher [26] and by 
Hofmann and Lewis [23]. Today the boundary regularity theory in the setting of uniformly elliptic and parabolic operators has reached a quite advanced level.

A natural geometrical setting for the uniformly parabolic operators in (1.12) is that of $\operatorname{Lip}(1,1 / 2)$ domains which we next introduce. We fix a $j \in\{1, \ldots, N\}$, we let $x^{\prime}=x_{j}$ and we let $x^{\prime \prime}$ denote the remaining $N-1$ coordinates of any $x \in \mathbb{R}^{N}$. We also let $e^{\prime}$ be the $j$-th vector of the canonical basis of $\mathbb{R}^{N}$, and we say that a function $f: \mathbb{R}^{N-1} \times \mathbb{R} \rightarrow \mathbb{R}$ is $\operatorname{Lip}(1,1 / 2)$, with respect to $x_{j}$, if

$$
\left|f\left(x^{\prime \prime}, t\right)-f\left(\xi^{\prime \prime}, \tau\right)\right| \leq M\left(\left|x^{\prime \prime}-\xi^{\prime \prime}\right|+|t-\tau|^{\frac{1}{2}}\right),
$$

for any $\left(x^{\prime \prime}, t\right),\left(\xi^{\prime \prime}, \tau\right) \in \mathbb{R}^{N-1} \times \mathbb{R}$, and for some positive constant $M$. For any $(x, t) \in \mathbb{R}^{N+1}$ and $r>0$ we set $\left.C_{r}(x, t)=B(x, r) \times\right] t-r^{2}, t+r^{2}$ [. Let $T$ be a positive number, let $\Omega \subset \mathbb{R}^{N+1}$ be a bounded domain. Let $\Omega_{T}=\Omega \cap\left(\mathbb{R}^{N} \times[0, T]\right)$, $S_{T}=\partial \Omega \cap\left(\mathbb{R}^{N} \times\right] 0, T[)$, and $\Delta(x, t, r)=S_{T} \cap C_{r}(x, t)$. Let $z=\left(x^{\prime}, x^{\prime \prime}, t\right) \in S_{T}$, with $x^{\prime}=x_{j}$ for some $j \in\{1, \ldots, N\}$. If there exist $r_{z}>0$ and a $\operatorname{Lip}(1,1 / 2)$ function $f$ with constant $M_{z}$, such that

$$
\begin{aligned}
& \Omega_{T} \cap C_{r_{z}}(z)=\left\{(\xi, \tau) \in \mathbb{R}^{N} \times[0, T] \mid \xi^{\prime}>f\left(\xi^{\prime \prime}, \tau\right)\right\} \cap C_{r_{z}}(z), \\
& S_{T} \cap C_{r_{z}}(z)=\left\{(\xi, \tau) \in \mathbb{R}^{N} \times[0, T] \mid \xi^{\prime}=f\left(\xi^{\prime \prime}, \tau\right)\right\} \cap C_{r_{z}}(z),
\end{aligned}
$$

then we say that $S_{T} \cap C_{r_{z}}(z)$ is $\operatorname{Lip}(1,1 / 2)$ surface, with constant $M_{z}$. If $S_{T}$ is covered by a finite set of cylinders $\left\{C_{r_{z_{j}}}\left(z_{i}\right)\right\}$, with $z_{i} \in S_{T}, r_{z_{i}}>0$, we set $M=\max _{i} M_{z_{i}}$ and $r_{0}=\min _{i} r_{z_{i}}$, and we say that $\Omega_{T}$ is a $\operatorname{Lip}(1,1 / 2)$ domain with constants $M$ and $r_{0}$.

Let $\Omega_{T}$ be a $\operatorname{Lip}(1,1 / 2)$ domain with constants $M$ and $r_{0}$, let $\left(x_{0}, t_{0}\right)$ be any point on $S_{T}$. For every $\left.r \in\right] 0, r_{0}$ [ with $t_{0}+r^{2}<T$, let $A_{r}^{+}\left(x_{0}, t_{0}\right)=\left(x_{0}+\right.$ $\left.2 M r e^{\prime}, t_{0}+r^{2}\right) \in \Omega$. Then

$$
M^{-1} r \leq d_{P}\left(A_{r}^{+}\left(x_{0}, t_{0}\right), S_{T}\right), \text { and } d_{P}\left(A_{r}^{+}\left(x_{0}, t_{0}\right),\left(x_{0}, t_{0}\right)\right)<r
$$

where $d_{P}$ denotes the standard parabolic distance function, $d_{P}((x, t),(y, s))=$ $|x-y|+|t-s|^{\frac{1}{2}}$ for any $(x, t),(y, s) \in \mathbb{R}^{N+1}$.

The following theorem is essentially due to Salsa, see [34, Theorem 3.1].

Let $\Omega_{T} \subset \mathbb{R}^{N+1}$ be a $\operatorname{Lip}(1,1 / 2)$ domain with constants $M$ and $r_{0}$, let $\left(x_{0}, t_{0}\right) \in S_{T}$ and assume that $r<\min \left\{r_{0} / 2, \sqrt{\left(T-t_{0}\right) / 4}, \sqrt{t_{0} / 4}\right\}$. Let $u$ be a non-negative solution to $L u=0$ in $\Omega_{T} \cap C_{2 r}\left(x_{0}, t_{0}\right)$ and assume that $u$ vanishes continuously on $\Delta\left(x_{0}, t_{0}, 2 r\right)$. Then there exists a constant $c=c\left(L, M, r_{0}\right), 1 \leq c<\infty$, such that

$$
u(x, t) \leq c u\left(A_{r}^{+}\left(x_{0}, t_{0}\right)\right)
$$

whenever $(x, t) \in \Omega_{T} \cap C_{r / c}\left(x_{0}, t_{0}\right)$.

While Salsa proved this theorem in the setting of time-independent Lipschitz cylinders, the proof goes through essentially unchanged in the more general setting of $\operatorname{Lip}(1,1 / 2)$ domains. This estimate is often referred to as a Carleson-type 
estimate, since this type of estimate first occurs in a paper by Carleson [3] on Fatou-type theorems for harmonic functions. Note that Theorem 1.1 is a generalization of this theorem. Indeed, in the Euclidean case we have $\left(x_{0}, t_{0}\right) \circ \delta_{r}(\tilde{x}, \widetilde{t})=$ $\left(x_{0}+r \tilde{x}, t_{0}+r^{2} \widetilde{t}\right)$, and we can choose $(\tilde{x}, \tilde{t})=\left(2 M e^{\prime}, 1\right)$ to recover the statement above. Furthermore, in the Euclidean case the notion of $\operatorname{Lip}_{K}$ surfaces introduced in Definition 2.1 below coincides with the notion of $\operatorname{Lip}(1,1 / 2)$ surfaces.

Returning to operators of Kolmogorov type $\mathscr{L}$, we note that if $\Sigma$ is a smooth subset of $\partial \Omega$ which is non-characteristic with respect to $\mathscr{L}$ according to (a) in Fichera's classification (1.11), then $\Sigma$ is $\operatorname{Lip}_{K}$. If we require less regularity on $\Sigma$, we may consider a surface $\Sigma \subset \partial \Omega$ which locally agrees with the graph $\left\{x_{j}=\right.$ $\left.f\left(x^{\prime \prime}, t\right)\right\}$ for some $\operatorname{Lip}(1,1 / 2)$ function $f$. In Proposition 2.2 below we prove that in this case $\Sigma$ is also a $\operatorname{Lip}_{K}$ surface. Hence Proposition 2.2 provides us with a simple sufficient condition for the $\operatorname{Lip}_{K}$ regularity. We also note that since $\mathscr{L}$ may be strongly degenerate, it is possible that a wide part $\Sigma$ of the boundary of a given open set $\Omega$ is characteristic and in this case $(b)$ in (1.11) is of interest. Indeed, when (b) is satisfied in some neighborhood $W \cap \Sigma$ of a point $z_{0} \in \partial \Omega$, then $W \cap \Sigma$ can be characterized as $t=g(x)$, where $g$ does not depend on $\left(x_{1}, \ldots, x_{m}\right)$. In this case, we in Proposition 2.4 below give a statement analogous to Theorem 1.1. Note that, in the case of uniformly parabolic operators, it turns out that $(b)$ is satisfied only by the lower basis of a cylinder.

The rest of the paper is organized as follows. Section 2 contains the definitions of $\operatorname{Lip}_{K}$ functions and $\operatorname{Lip}_{K}$ surfaces, and the statement of the key propositions established in order to prove our main result, Theorem 1.1. Section 3 is of preliminary nature and we here collect some notions and we state a few results concerning the interior Harnack inequality. In Section 4 we prove a few basic boundary estimates for non-negative solutions to $\mathscr{L} u=0$ near Lip ${ }_{K}$ surfaces. Section 5 is devoted to the proof of Theorem 1.1, and to the proof of the propositions stated in Section 2.

Acknowledgements. We thank E. Lanconelli, F. Ferrari and S. Salsa for their interest in our work.

\section{2. $\operatorname{Lip}_{K}$ surfaces and statement of key propositions}

In the sequel we will write the dilation (1.8) in the following form

$$
\delta_{r}=\operatorname{diag}\left(r^{\alpha_{1}}, \ldots, r^{\alpha_{N}}, r^{2}\right)
$$

where we set $\alpha_{1}=\ldots=\alpha_{m}=1$, and $\alpha_{m+m_{1}+\cdots+m_{j-1}+1}=\ldots=\alpha_{m+m_{1}+\cdots+m_{j}+1}=$ $2 j+1$ for $j=1, \ldots, \kappa$. According to (1.8), we split the coordinate $x \in \mathbb{R}^{N}$ as

$$
x=\left(x^{(0)}, x^{(1)}, \ldots, x^{(\kappa)}\right), \quad x^{(0)} \in \mathbb{R}^{m}, \quad x^{(j)} \in \mathbb{R}^{m_{j}}, \quad j \in\{1, \ldots, \kappa\},
$$


and we define

$$
|x|_{K}=\sum_{j=0}^{\kappa}\left|x^{(j)}\right|^{\frac{1}{2 j+1}}, \quad\|(x, t)\|_{K}=|x|_{K}+|t|^{\frac{1}{2}}
$$

Note that $\left\|\delta_{r} z\right\|_{K}=r\|z\|_{K}$ for every $r>0$ and $z \in \mathbb{R}^{N+1}$. We recall the following pseudo-triangular inequality: there exists a positive constant $\mathbf{c}$ such that

$$
\left\|z^{-1}\right\|_{K} \leq \mathbf{c}\|z\|_{K}, \quad\|z \circ \zeta\|_{K} \leq \mathbf{c}\left(\|z\|_{K}+\|\zeta\|_{K}\right), \quad z, \zeta \in \mathbb{R}^{N+1} .
$$

We also define the quasi-distance $d_{K}$ by setting

$$
d_{K}(z, \zeta):=\left\|\zeta^{-1} \circ z\right\|_{K}, \quad z, \zeta \in \mathbb{R}^{N+1},
$$

and the ball

$$
\mathcal{B}_{K}\left(z_{0}, r\right):=\left\{z \in \mathbb{R}^{N+1} \mid d_{K}\left(z, z_{0}\right)<r\right\} .
$$

Note that from (2.3) it directly follows

$$
d_{K}(z, \zeta) \leq \mathbf{c}\left(d_{K}(z, w)+d_{K}(w, \zeta)\right), \quad z, \zeta, w \in \mathbb{R}^{N+1}
$$

For any $z \in \mathbb{R}^{N+1}$ and $H \subset \mathbb{R}^{N+1}$, we define

$$
d_{K}(z, H):=\inf \left\{d_{K}(z, \zeta) \mid \zeta \in H\right\}
$$

We say that a function $f: \Omega \rightarrow \mathbb{R}$ is Hölder continuous of exponent $\alpha \in] 0,1]$, in short $f \in C_{K}^{0, \alpha}(\Omega)$, if there exists a positive constant $C$ such that

$$
|f(z)-f(\zeta)| \leq C d_{K}(z, \zeta)^{\alpha}, \quad \text { for every } z, \zeta \in \Omega
$$

We next define $\operatorname{Lip}_{K}$ functions and $\operatorname{Lip}_{K}$ surfaces. For any given $x \in \mathbb{R}^{N}$ we set

$$
x^{\prime}=x_{1} \quad \text { and } \quad x^{\prime \prime}=\left(x_{2}, \ldots, x_{N}\right) .
$$

Moreover, using the notation in (2.2) we also let $x_{\prime}^{(0)}=x_{1}=x^{\prime}$, and we let $x_{\prime \prime}^{(0)}=$ $\left(x_{2}, \ldots, x_{m}\right)$. Using this notation, we define the norm

$$
\left\|\left(x^{\prime \prime}, t\right)\right\|_{K}^{\prime \prime}=\left|x_{\prime \prime}^{(0)}\right|+\sum_{j=1}^{\kappa}\left|x^{(j)}\right|^{\frac{1}{2 j+1}}+|t|^{\frac{1}{2}}
$$

in $\mathbb{R}^{N-1} \times \mathbb{R}$. Recalling (1.8), we let

$$
D_{r}^{\prime \prime}=\operatorname{diag}\left(r I_{m-1}, r^{3} I_{m_{1}}, \ldots, r^{2 \kappa+1} I_{m_{\kappa}}\right),
$$


so that $D_{r}^{\prime \prime} x^{\prime \prime}=\left(D_{r} x\right)^{\prime \prime}$, while $\left(D_{r} x\right)^{\prime}=r x^{\prime}$. Note that $\left\|\left(D_{r}^{\prime \prime} x^{\prime \prime}, r^{2} t\right)\right\|_{K}^{\prime \prime}=$ $r\left\|\left(x^{\prime \prime}, t\right)\right\|_{K}^{\prime \prime}$ for every $r>0$ and $\left(x^{\prime \prime}, t\right) \in \mathbb{R}^{N-1} \times \mathbb{R}$. Using the notation in (2.1) we set, for any positive $r_{1}, r_{2}, r_{3}$,

$$
\begin{aligned}
Q_{r_{1}, r_{2}, r_{3}} & =\left\{(x, t) \in \mathbb{R}^{N+1}|| x^{\prime}\left|\leq r_{1},\right| x_{i} \mid \leq r_{2}^{\alpha_{i}} \text { for any } i=2, \ldots, N,|t| \leq r_{3}^{2}\right\}, \\
Q_{r_{2}, r_{3}}^{\prime \prime} & =\left\{\left(x^{\prime \prime}, t\right) \in \mathbb{R}^{N-1} \times \mathbb{R}|| x_{i} \mid \leq r_{2}^{\alpha_{i}} \text { for any } i=2, \ldots, N,|t| \leq r_{3}^{2}\right\},
\end{aligned}
$$

and, for any positive $M$ and $r$ and any arbitrary point $z_{0} \in \mathbb{R}^{N+1}$, we define

$$
Q_{M, r}=Q_{4 M r, r, \sqrt{2} r}, \quad Q_{r}^{\prime \prime}=Q_{r, \sqrt{2} r}^{\prime \prime}, \quad Q_{M, r}\left(z_{0}\right)=z_{0} \circ Q_{M, r} .
$$

Note that $Q_{M, r}=\delta_{r} Q_{M, 1}$ and $Q_{r}^{\prime \prime}=D_{r}^{\prime \prime} Q_{1}^{\prime \prime}$ for every $r>0$. Moreover, the continuity of the group law (1.5) implies that there exists $\varepsilon=\varepsilon(\mathscr{L}, M) \in] 0,1[$ such that

$$
(x, t) \circ(\xi, \tau) \in Q_{M, r} \quad \forall(\xi, \tau) \in Q_{M, \varepsilon r}, \quad \forall(x, t) \in Q_{M, \frac{r}{2}} .
$$

Furthermore, for every positive $M$, there exist two positive constants $c_{M}^{\prime}, c_{M}^{\prime \prime}$ such that

$$
\mathcal{B}_{K}\left(z_{0}, c_{M}^{\prime} r\right) \subseteq Q_{M, r}\left(z_{0}\right) \subseteq \mathcal{B}_{K}\left(z_{0}, c_{M}^{\prime \prime} r\right)
$$

for every $z_{0} \in \mathbb{R}^{N+1}$ and $r>0$.

Let $e^{\prime} \in \mathbb{R}^{m}$ be such that $\left\|e^{\prime}\right\|=1$. Since the form of the matrix $B$ in (1.7) remains invariant under rotation in the first $m$ variables it is not restrictive to assume $e^{\prime}=e_{1}=(1,0, \ldots, 0)$. Given any open set $\Omega^{\prime \prime} \subset \mathbb{R}^{N-1} \times \mathbb{R}$, we say that $f: \Omega^{\prime \prime} \rightarrow$ $\mathbb{R}$ is a $\operatorname{Lip}_{K}$ function with respect to $e^{\prime}$, if $x^{\prime}=x_{1}$ and

$$
\left|f\left(\left(x+\exp \left(-t B^{T}\right) x_{0}\right)^{\prime \prime}, t+t_{0}\right)-f\left(x_{0}^{\prime \prime}, t_{0}\right)\right| \leq M\left\|\left(x^{\prime \prime}, t\right)\right\|_{K}^{\prime \prime},
$$

for every $\left(x_{0}^{\prime \prime}, t_{0}\right) \in \Omega^{\prime \prime}$, and $\left(x^{\prime \prime}, t\right) \in \mathbb{R}^{N-1} \times \mathbb{R}$ such that $\left(\left(x+\exp \left(-t B^{T}\right) x_{0}\right)^{\prime \prime}, t+\right.$ $\left.t_{0}\right) \in \Omega^{\prime \prime}, x_{0}^{\prime}=f\left(x_{0}^{\prime \prime}, t_{0}\right)$. Note that we can assume, without loss of generality, that $\left(x_{0}, t_{0}\right)=(0,0)$ and $f(0,0)=0$. Indeed, if necessary, it is enough to set $g\left(x^{\prime \prime}, t\right)=f\left(\left(x+\exp \left(-t B^{T}\right) x_{0}\right)^{\prime \prime}, t+t_{0}\right)-f\left(x_{0}^{\prime \prime}, t_{0}\right)$. Equivalently, $f: \Omega^{\prime \prime} \rightarrow \mathbb{R}$ is $\operatorname{Lip}_{K}$ if

$$
\left|f\left(x^{\prime \prime}, t\right)-f\left(\xi^{\prime \prime}, \tau\right)\right| \leq M\left\|\left(\left(x-\exp \left((\tau-t) B^{T}\right) \xi\right)^{\prime \prime}, t-\tau\right)\right\|_{K}^{\prime \prime},
$$

for every $\left(x^{\prime \prime}, t\right),\left(\xi^{\prime \prime}, \tau\right) \in \Omega^{\prime \prime}, x^{\prime}=f\left(x^{\prime \prime}, t\right), \xi^{\prime}=f\left(\xi^{\prime \prime}, \tau\right)$. Given $f$ as above with $f(0,0)=0$ and $M, r>0$, we define

$$
\Omega_{f, r}=\left\{(x, t) \in Q_{M, r} \mid f\left(x^{\prime \prime}, t\right)<x^{\prime}\right\}, \quad \Delta_{f, r}=\left\{(x, t) \in Q_{M, r} \mid f\left(x^{\prime \prime}, t\right)=x^{\prime}\right\} .
$$

Note that, according to the dilations $\delta_{r}$ and $D_{r}^{\prime \prime}$, we have

$$
\Omega_{f, r}=\delta_{r} \Omega_{f_{r}, 1}, \quad \Delta_{f, r}=\delta_{r} \Delta_{f_{r}, 1},
$$


where $f_{r}\left(x^{\prime \prime}, t\right)=r^{-1} f\left(D_{r}^{\prime \prime} x^{\prime \prime}, r^{2} t\right)$. We point out that if $f$ is a $\operatorname{Lip}_{K}$ function, then so is $f_{r}$. Indeed, as the norm $\|\cdot\|_{K}^{\prime \prime}$ is $\left(D_{r}^{\prime \prime}, r^{2}\right)$-homogeneous, we have

$$
\left|f_{r}\left(y^{\prime \prime}, s\right)-f_{r}\left(x_{0}^{\prime \prime}, t_{0}\right)\right| \leq M\left\|\left(\left(y-\exp \left(\left(t_{0}-s\right) B^{T}\right) x_{0}\right)^{\prime \prime}, s-t_{0}\right)\right\|_{K}^{\prime \prime},
$$

for every $\left(y^{\prime \prime}, s\right),\left(x_{0}^{\prime \prime}, t_{0}\right) \in\left(D_{r}^{\prime \prime}, r^{2}\right)^{-1} \Omega^{\prime \prime}$ and $r>0$. Finally, we let

$$
\Omega_{f, r}\left(z_{0}\right)=z_{0} \circ \Omega_{f, r}, \quad \Delta_{f, r}\left(z_{0}\right)=z_{0} \circ \Delta_{f, r}, \quad z_{0} \in \mathbb{R}^{N+1} .
$$

Definition 2.1. Let $\Omega \subset \mathbb{R}^{N+1}$ be a bounded domain. We say that $\Sigma \subset \partial \Omega$ is a $\operatorname{Lip}_{K}$ surface with constants $M=\max \left\{M_{1}, \ldots, M_{k}\right\}$ and $r_{0}=\min \left\{r_{1}, \ldots, r_{k}\right\}$ if, for any $j=1, \ldots, k$, there exists a point $z_{j} \in \Sigma$, and a $\operatorname{Lip}_{K}$ function $f_{j}: Q_{2 r_{j}}^{\prime \prime} \rightarrow$ $\mathbb{R}$ defined with respect to a suitable $e_{j}^{\prime} \in \mathbb{R}^{m}$ and with Lipschitz constant $M_{j}$, such that

$$
\begin{aligned}
& \Sigma \subset \bigcup_{j=1}^{k} \Delta_{f_{j}, r_{j}}\left(z_{j}\right), \\
& \Sigma \cap Q_{M_{j}, 2 r_{j}}\left(z_{j}\right)=\Delta_{f_{j}, 2 r_{j}}\left(z_{j}\right), \\
& \Omega \cap Q_{M_{j}, 2 r_{j}}\left(z_{j}\right)=\Omega_{f_{j}, 2 r_{j}}\left(z_{j}\right) .
\end{aligned}
$$

Recall the definition of $\operatorname{Lip}(1,1 / 2)$ functions given in (1.13). For the proof of the following proposition we refer to Section 5 .

Proposition 2.2. Let $\Omega \subset \mathbb{R}^{N+1}$ be a bounded domain. If $\Sigma \subset \partial \Omega$ locally agrees with the graph $\left\{x_{j}=f\left(x^{\prime \prime}, t\right)\right\}$ of some $\operatorname{Lip}(1,1 / 2)$ function $f, j \in\{1, \ldots, m\}$, then $\Sigma$ is a $\operatorname{Lip}_{K}$ surface.

The following proposition plays a key role in the proof of Theorem 1.1 and will be proved in Section 5.

Proposition 2.3. Let $Q_{r}^{\prime \prime}$ be as in (2.10) with $\left.\left.r \in\right] 0,1\right]$. Let $f: Q_{r}^{\prime \prime} \rightarrow \mathbb{R}$ be a $\operatorname{Lip}_{K}$ function such that $f(0,0)=0$. Then there exist a point $(\tilde{x}, \widetilde{t}) \in \mathbb{R}^{N+1}$ and two positive constants $C, c$, depending only on $\mathscr{L}$ and $M$, such that

$$
\sup _{\Omega_{f, c r}\left(x_{0}, t_{0}\right)} u(x, t) \leq C u\left(A_{r}^{+}\left(x_{0}, t_{0}\right)\right), \quad A_{r}^{+}\left(x_{0}, t_{0}\right)=\left(x_{0}, t_{0}\right) \circ \delta_{r}(\tilde{x}, \tilde{t}),
$$

for every positive solution $u$ to $\mathscr{L} u=0$ in $\Omega_{f, r}\left(x_{0}, t_{0}\right)$ vanishing continuously on $\Delta_{f, r}\left(x_{0}, t_{0}\right)$.

We next formulate two versions of Proposition 2.3 which apply to characteristic boundary points with respect to $\mathscr{L}$. In particular, Proposition 2.4 below applies to a cylinder $\widetilde{\Omega}_{g, r}\left(z_{0}\right)=z_{0} \circ \widetilde{\Omega}_{g, r}$ obtained by using the operation "o", while in Proposition 2.5 the operation "o" does not appear. Note that, in the latter case, the Lipschitz constant $M$ of the function $g$ depends on the cylinder. To proceed, let

$$
\widetilde{Q}_{r}=\left\{\left(x^{(1)}, \ldots, x^{(\kappa)}\right) \in \mathbb{R}^{N-m}|| x^{(j)} \mid \leq r^{\frac{1}{2 j+1}}, j=1, \ldots, \kappa\right\} .
$$


Let $g: \widetilde{Q}_{r} \rightarrow \mathbb{R}$ be a Lipschitz function in the classical sense, with Lipschitz constant $M$, i.e.

$\left|g\left(x^{(1)}, \ldots, x^{(\kappa)}\right)-g\left(y^{(1)}, \ldots, y^{(\kappa)}\right)\right| \leq M\left(\left|x^{(1)}-y^{(1)}\right|+\ldots .+\left|x^{(\kappa)}-y^{(\kappa)}\right|\right)$

whenever $\left(x^{(1)}, \ldots, x^{(\kappa)}\right),\left(y^{(1)}, \ldots, y^{(\kappa)}\right) \in \widetilde{Q}_{r}$. Furthermore, assume $g(0)=0$. Then, for positive $r$ and $z_{0} \in \mathbb{R}^{N+1}$, we define

$$
\begin{aligned}
& \widetilde{\Omega}_{g, r}=\left\{(x, t) \in Q_{r, r, M r} \mid t>g\left(x^{(1)}, \ldots, x^{(\kappa)}\right)\right\}, \\
& \widetilde{\Delta}_{g, r}=\left\{(x, t) \in Q_{r, r, M r} \mid t=g\left(x^{(1)}, \ldots, x^{(\kappa)}\right)\right\}, \\
& \widetilde{\Omega}_{g, r}\left(z_{0}\right)=z_{0} \circ \widetilde{\Omega}_{g, r}, \quad \widetilde{\Delta}_{g, r}\left(z_{0}\right)=z_{0} \circ \widetilde{\Delta}_{g, r} .
\end{aligned}
$$

Proposition 2.4. Let $\widetilde{Q}_{r}$ be as in (2.16) with $\left.\left.r \in\right] 0,1\right]$. Let $g: \widetilde{Q}_{r} \rightarrow \mathbb{R}$ be a Lipschitz function with Lipschitz constant $M$ such that $g(0)=0$. Then there exist three positive constants $C, c$ and $\tilde{t}$, depending only on $\mathscr{L}$ and $M$, such that

$$
\sup _{\widetilde{\Omega}_{g, c r}\left(x_{0}, t_{0}\right)} u(x, t) \leq C u\left(A_{r}^{+}\left(x_{0}, t_{0}\right)\right), \quad A_{r}^{+}\left(x_{0}, t_{0}\right)=\left(x_{0}, t_{0}\right) \circ\left(0, r^{2} \widetilde{t}\right),
$$

for every positive solution $u$ to $\mathscr{L} u=0$ in $\widetilde{\Omega}_{g, r}\left(x_{0}, t_{0}\right)$ vanishing continuously on $\widetilde{\Delta}_{g, r}\left(x_{0}, t_{0}\right)$.

Let $Q$ be a bounded set of $\mathbb{R}^{N}$, and let $g: Q \rightarrow \mathbb{R}$ be a Lipschitz function, which does not depend on $x_{1}, \ldots, x_{m}$, with constant $M$, i.e. $g(x)=g\left(x^{(1)}, \ldots, x^{(\kappa)}\right)$. Letting $g\left(x_{0}\right)=t_{0}$ for some $x_{0} \in Q$, and $c_{Q}=\sup _{x \in Q}\left|x-x_{0}\right|$, we define

$$
\begin{aligned}
& \widetilde{\Omega}_{g}=\left\{(x, t) \in \mathbb{R}^{N+1} \mid x \in Q, g(x)<t \leq t_{0}+M c_{Q}\right\}, \\
& \widetilde{\Delta}_{g}=\left\{(x, t) \in \mathbb{R}^{N+1} \mid x \in Q, t=g(x)\right\} .
\end{aligned}
$$

Proposition 2.5. Let $x_{0} \in \mathbb{R}^{N}$, let $Q$ be a bounded neighborhood of $x_{0}$, and let $M$ be a positive constant such that $M \sup _{x \in O}\left\|B^{T} x\right\|<1$. Let $g: Q \rightarrow \mathbb{R}$ be a Lipschitz function, which does not depend on $x_{1}, \ldots, x_{m}$, with constant $M$ and let $t_{0}=g\left(x_{0}\right)$. Then there exist three positive constants $C, r$ and $\tilde{t}$, only depending on $\mathscr{L}$ and $Q$, such that

$$
\sup _{\widetilde{\Omega}_{g} \cap \mathcal{B}_{K}\left(\left(x_{0}, t_{0}\right), r\right)} u(x, t) \leq C u\left(A_{r}^{+}\left(x_{0}, t_{0}\right)\right), \quad A_{r}^{+}\left(x_{0}, t_{0}\right)=\left(x_{0}, t_{0}\right) \circ\left(0, r^{2} \widetilde{t}\right),
$$

for every positive solution u to $\mathscr{L} u=0$ in $\widetilde{\Omega}_{g}$ vanishing continuously on $\widetilde{\Delta}_{g}$.

The proofs of Proposition 2.4 and Proposition 2.5 are given in Section 5. 


\section{Interior Harnack inequalities}

We say that a path $\gamma:[0, T] \rightarrow \mathbb{R}^{N+1}$ is $\mathscr{L}$-admissible if it is absolutely continuous and satisfies

$$
\gamma^{\prime}(s)=\sum_{j=1}^{m} \omega_{j}(s) X_{j}(\gamma(s))+\lambda(s) Y(\gamma(s)), \quad \text { for a.e. } s \in[0, T],
$$

where $\omega=\left(\omega_{1}, \ldots, \omega_{m}\right) \in L^{2}\left([0, T], \mathbb{R}^{m}\right)$, and $\lambda$ is a strictly positive measurable function. We say that $\gamma$ steers $z_{0}$ to $z$ if $\gamma(0)=z_{0}$ and $\gamma(T)=z$. Concerning the problem of the existence of admissible paths, we recall that it is a controllability problem, and that [H.2] is equivalent to the following Kalman condition:

$$
\operatorname{rank}\left(\bar{A} B^{T} \bar{A} \cdots\left(B^{T}\right)^{N-1} \bar{A}\right)=N .
$$

Here $\bar{A}$ is the $N \times N$ matrix defined by

$$
\left(\begin{array}{cc}
\bar{A}_{0} & 0 \\
0 & 0
\end{array}\right)
$$

and $\bar{A}_{0}$ is the $m \times m$ constant matrix introduced in (1.3). We recall that (3.2) is a sufficient condition for the global controllability of (3.1), i.e. the property that any point $z_{0}=\left(x_{0}, t_{0}\right) \in \mathbb{R}^{N+1}$ can be connected to any $z=(x, t) \in \mathbb{R}^{N+1}$ with $t<t_{0}$ by an $\mathscr{L}$-admissible path (see [29], Theorem 5, page 81 ). In the sequel we let $A_{z_{0}}(\Omega)=\left\{z \in \Omega \mid\right.$ there exists an $\mathscr{L}$-admissible $\gamma:[0, T] \rightarrow \Omega$ connecting $z_{0}$ to $\left.z\right\}$, and we define $\mathscr{A}_{z_{0}}=\mathscr{A}_{z_{0}}(\Omega)=\overline{A_{z_{0}}(\Omega)}$ as the closure (in $\mathbb{R}^{N+1}$ ) of $A_{z_{0}}(\Omega)$. We will refer to the set $\mathscr{A}_{z_{0}}$ as the attainable set.

We next recall a Harnack type inequality which is stated in terms of $\mathscr{L}$-admissible paths and attainable sets.

Theorem 3.1. (THEOREM 2.4 IN [6]) Let $\mathscr{L}$ be an operator in the form (1.1), satisfying assumptions [H.1-3]. Let $\Omega$ be an open subset of $\mathbb{R}^{N+1}$ and let $z_{0} \in \Omega$. For every compact set $H \subseteq \operatorname{Int}\left(\mathscr{A}_{z_{0}}\right)$, there exists a positive constant $C_{H}$, only dependent on $\Omega, z_{0}, H$ and on the operator $\mathscr{L}$, such that

$$
\sup _{H} u \leq C_{H} u\left(z_{0}\right),
$$

for every non-negative solution $u$ of $\mathscr{L} u=0$ in $\Omega$.

The following Proposition 3.2 is a consequence of [11, Theorem 1.2] and [11, Lemma 6.2]. We give here a simple proof based on our Theorem 3.1. For any positive $r, \beta$ and $\left(x_{0}, t_{0}\right) \in \mathbb{R}^{N+1}$, according to notation (2.9) we set

$$
\begin{array}{ll}
Q_{r}^{-}=Q_{r, r, r} \cap\left\{(x, t) \in \mathbb{R}^{N+1} \mid t<0\right\}, & Q_{r}^{-}\left(x_{0}, t_{0}\right)=\left(x_{0}, t_{0}\right) \circ Q_{r}^{-}, \\
K_{\beta r}=Q_{\beta r, \beta r, r} \cap\left\{(x, t) \in \mathbb{R}^{N+1} \mid t=-r^{2} / 2\right\}, & K_{\beta r}\left(x_{0}, t_{0}\right)=\left(x_{0}, t_{0}\right) \circ K_{\beta r} .
\end{array}
$$


Proposition 3.2. Let $\gamma$ be an $\mathscr{L}$-admissible path satisfying $\gamma(0)=\left(x_{0}, t_{0}\right)$. There exist two positive constants $h$ and $C$ such that

$$
\int_{0}^{s}|\omega(\tau)|^{2} d \tau \leq h \quad \Rightarrow \quad u(\gamma(s)) \leq C u\left(x_{0}, t_{0}\right)
$$

for every non-negative solution $u$ of $\mathscr{L} u=0$ in $Q_{1}^{-}\left(x_{0}, t_{0}\right)$ and $\left.\left.s \in\right] 0,1 / 2\right]$.

Proof. We first claim that there exists $\beta \in] 0,1\left[\right.$ such that $K_{\beta r}\left(x_{0}, t_{0}\right)$ is contained in $\operatorname{Int}\left(\mathscr{A}_{\left(x_{0}, t_{0}\right)}\left(Q_{r}^{-}\left(x_{0}, t_{0}\right)\right)\right)$. To check the above statement, it is sufficient to consider the case $\left(x_{0}, t_{0}\right)=(0,0)$ and $r=1$. We first note that, as $\omega_{1} \equiv 0, \ldots, \omega_{m} \equiv 0$, we have $\gamma(s)=(0,-s)$, then $(0,-1 / 2) \in A_{(0,0)}\left(Q_{1}^{-}\right)$. To prove that $(0,-1 / 2)$ is an interior point of $\mathscr{A}_{(0,0)}\left(Q_{1}^{-}\right)$, we recall that the system

$$
\gamma^{\prime}(s)=\sum_{j=1}^{m} \omega_{j}(s) X_{j}(\gamma(s))+Y(\gamma(s)), \quad \gamma(0)=(0,0),
$$

is globally controllable, by (3.2). Then, as $\omega$ varies in a neighborhood of the vector $0 \in L^{2}\left([0, T], \mathbb{R}^{m}\right)$, the image of the map $\omega \mapsto \gamma(T)$ covers a neighborhood of $(0,-1 / 2)$, and $\gamma(s) \in Q_{1}^{-}$for every $s \in[0, T]$. This proves the claim.

Let $\beta$ be as above. By Theorem 3.1, there exists a positive constant $C_{\beta}$ such that

$$
\sup _{K_{\beta r}\left(x_{0}, t_{0}\right)} u \leq C_{\beta} u\left(x_{0}, t_{0}\right),
$$

for every non-negative solution $u$ of $\mathscr{L} u=0$ in $Q_{1}^{-}\left(x_{0}, t_{0}\right)$ and for any $\left.\left.r \in\right] 0,1\right]$. Finally, a plain application of the Hölder inequality shows that

$$
\left.\left.\gamma(s) \in K_{\beta r}\left(x_{0}, t_{0}\right) \quad \text { whenever } \quad s \in\right] 0,1 / 2\right] \quad \text { satisfies } \quad \int_{0}^{s}|\omega(\tau)|^{2} d \tau \leq h,
$$

for a suitable constant $h$. We refer to [11, Lemma 6.2] for more details. The conclusion of the proof easily follows from (3.7) and (3.6).

\section{Basic boundary estimates}

We first introduce a family of cones defined in terms of the dilation $\delta_{\lambda}$ and the translation "o". For any given $z_{0} \in \mathbb{R}^{N+1}, \bar{x} \in \mathbb{R}^{N}, \bar{t} \in \mathbb{R}^{+}$, we consider an open neighborhood $U \subset \mathbb{R}^{N}$ of $\bar{x}$, and we denote by $Z_{\bar{x}, \bar{t}, U}^{-}\left(z_{0}\right)$ and $Z_{\bar{x}, \bar{t}, U}^{+}\left(z_{0}\right)$ the following tusk-shaped sets

$$
\begin{aligned}
& Z_{\bar{x}, \bar{t}, U}^{-}\left(z_{0}\right)=\left\{z_{0} \circ \delta_{s}(x,-\bar{t}) \mid x \in U, 0<s \leq 1\right\}, \\
& Z_{\bar{x}, \bar{t}, U}^{+}\left(z_{0}\right)=\left\{z_{0} \circ \delta_{s}(x, \bar{t}) \mid x \in U, 0<s \leq 1\right\} .
\end{aligned}
$$


In the sequel, aiming to simplify the notations, we will often write $Z^{ \pm}\left(z_{0}\right)$ instead of $Z_{\bar{x}, \bar{t}, U}^{ \pm}\left(z_{0}\right)$ if the choice of $\bar{x}, \bar{t}, U$ is clear from the context. Note that $Z^{-}\left(z_{0}\right)$ and $Z^{+}\left(z_{0}\right)$ are cones with the same vertex at $z_{0}=\left(x_{0}, t_{0}\right)$, while the basis of $Z^{-}\left(z_{0}\right)$ is at the time level $t_{0}-\bar{t}<t_{0}$, and the basis of $Z^{+}\left(z_{0}\right)$ is at the time level $t_{0}+\bar{t}>t_{0}$.

Definition 4.1. Let $\Omega$ be an open subset of $\mathbb{R}^{N+1}$ and let $\Sigma \subset \partial \Omega$.

(i) We say that $\Sigma$ satisfies an uniform exterior cone condition if there exist $\bar{x} \in$ $\mathbb{R}^{N}, \bar{t}>0$ and an open neighborhood $U \subseteq \mathbb{R}^{N}$ of $\bar{x}$ such that

$$
Z^{-}\left(z_{0}\right) \cap \Omega=\emptyset \quad \text { for every } \quad z_{0} \in \Sigma,
$$

where $Z^{-}\left(z_{0}\right)=Z_{\bar{x}, \bar{t}, U}^{-}\left(z_{0}\right)$.

(ii) We say that $\Sigma$ satisfies an uniform interior cone condition if there exist $\bar{x} \in$ $\mathbb{R}^{N}, \bar{t}>0$ and an open neighborhood $U \subseteq \mathbb{R}^{N}$ of $\bar{x}$ such that

$$
Z^{+}\left(z_{0}\right) \subset \Omega \quad \text { for every } z_{0} \in \Sigma,
$$

where $Z^{+}\left(z_{0}\right)=Z_{\bar{x}, \bar{t}, U}^{+}\left(z_{0}\right)$.

(iii) Assume that $\Sigma$ satisfies an uniform interior cone condition in the sense stated above. We then say that the cones $\left\{Z^{+}\left(z_{0}\right)\right\}=\left\{Z_{\bar{x}, \bar{t}, U}^{+}\left(z_{0}\right)\right\}, z_{0} \in \Sigma$, satisfy a strong Harnack connectivity condition if the function $s \mapsto\left(x_{0}, t_{0}\right) \circ \delta_{1-s}(\bar{x}, \bar{t})$ is an $\mathscr{L}$-admissible path.

We point out that the strong Harnack connectivity condition is more restrictive than the Harnack connectivity condition used in our previous work [6]. Nevertheless, we are able to prove the validity of this condition near $\operatorname{Lip}_{K}$ surfaces.

We next show how to find a point $x_{\Lambda} \in \mathbb{R}^{N}$ such that the path $s \mapsto \delta_{1-s}\left(x_{\Lambda}, 1\right)$ is $\mathscr{L}$-admissible. To this aim, we recall notations (1.7) and (2.2).

Lemma 4.2. For any positive $\Lambda$ we define the point $x_{\Lambda} \in \mathbb{R}^{N}$ as follows:

$$
x_{\Lambda}^{(0)}=\Lambda e^{\prime}, \quad x_{\Lambda}^{(j)}=-\frac{2}{2 j+1} B_{j}^{T} x_{\Lambda}^{(j-1)}, \quad j=1, \ldots, \kappa .
$$

Here $e^{\prime}$ is the unit vector of $\mathbb{R}^{m}$ pointing towards the $x^{\prime}$-direction. Then, the path $[0,1] \ni s \rightarrow \gamma(s)=\delta_{1-s}\left(x_{\Lambda}, 1\right)$ is $\mathscr{L}$-admissible.

Proof. We show that $\gamma$ satisfies (3.1), namely

$$
\gamma^{\prime}(s)=\bar{A}_{0} \omega(s)+\lambda(s)\left(B^{T} \gamma(s)-\partial_{t}\right) \quad \text { a.e. in }[0,1],
$$

for a $\omega \in L^{2}\left([0,1], \mathbb{R}^{m}\right)$ and a positive measurable function $\lambda$. By a direct computation,

$$
\begin{aligned}
\gamma(s)=\left((1-s) x_{\Lambda}^{(0)},\right. & -\frac{2}{3}(1-s)^{3} B_{1}^{T} x_{\Lambda}^{(0)}, \ldots \\
& \left.\frac{(-2)^{\kappa}}{(2 \kappa+1) ! !}(1-s)^{2 \kappa+1} B_{\kappa}^{T} \cdots B_{1}^{T} x_{\Lambda}^{(0)},(1-s)^{2}\right)
\end{aligned}
$$


so that we find

$$
\gamma^{\prime}(s)=\left(-x_{\Lambda}^{(0)}, 0, \ldots, 0\right)+2(1-s)\left(B^{T} \gamma(s)-\partial_{t}\right), \quad s \in[0,1] .
$$

This proves (4.3) with $\omega=-\bar{A}_{0}^{-1} x_{\Lambda}^{(0)}$ and $\lambda(s)=2(1-s)$.

Our next result improves on the analogous one in [6, Proposition 3.2]. Indeed, as noticed in [6, Remark 4.2], it fails under the weaker Harnack connectivity condition assumed in [6].

Lemma 4.3. Let $Z_{\bar{x}, \bar{t}, U}^{+}(0,0)$ be a cone satisfying the strong Harnack connectivity condition (iii) in Definition 4.1. Then there exist two positive constants $C_{1}$ and $\beta$, which only depend on $Z^{+}$and on the operator $\mathscr{L}$, such that

$$
u\left(\delta_{s}(\bar{x}, \bar{t})\right) \leq \frac{C_{1}}{\left\|\delta_{s}(\bar{x}, \bar{t})\right\|_{K}^{\beta}} u(\bar{x}, \bar{t}) \quad 0<s<1,
$$

for every non-negative solution u of $\mathscr{L} u=0$ in $Z^{+}$.

Proof. We first show that there exist a positive constant $\widetilde{C}$ and $\left.s_{0} \in\right] 0,1[$ such that

$$
u\left(\delta_{\sigma}(\bar{x}, \bar{t})\right) \leq \widetilde{C} u(\bar{x}, \bar{t}), \quad \text { for every } \sigma \in\left[1-s_{0}, 1[\right.
$$

To this aim, we note that there exists $\rho \in] 0,1]$ such that $Q_{\rho}^{-}(\bar{x}, \bar{t}) \subset Z_{\bar{x}, \bar{t}, U}^{+}(0,0)$. Moreover, the path $\gamma(s)=\delta_{1-s}(\bar{x}, \bar{t})$ is $\mathscr{L}$-admissible by the strong Harnack connectivity condition. Since $\omega_{1}, \ldots, \omega_{m} \in L^{2}([0,1])$, there exists $\left.s_{0} \in\right] 0,1[$ such that

$$
\int_{0}^{s_{0}}|\omega(\tau)|^{2} d \tau \leq h
$$

where $h$ is the positive constant appearing in Proposition 3.2. Then (4.4) directly follows from Proposition 3.2.

We next conclude the proof by applying several times (4.4). For a given $s \in$ ] $0,1-s_{0}\left[\right.$, we set $\widetilde{Z}^{+}(0,0)=\delta_{\left(1-s_{0}\right) / s}\left(Z^{+}(0,0)\right)$. Note that the function

$$
u_{s}: \widetilde{Z}^{+}(0,0) \rightarrow \mathbb{R}, \quad u_{s}=u\left(\delta_{s /\left(1-s_{0}\right)}(\cdot)\right)
$$

is a non-negative solution to $\mathscr{L}_{s} u_{s}=0$, where

$$
\begin{aligned}
\mathscr{L}_{s}= & \sum_{i, j=1}^{m} a_{i, j}\left(\delta_{s /\left(1-s_{0}\right)}(z)\right) \partial_{x_{i} x_{j}}+\sum_{i=1}^{m} \frac{s}{\left(1-s_{0}\right)} a_{i}\left(\delta_{s /\left(1-s_{0}\right)}(z)\right) \partial_{x_{i}} \\
& +\sum_{i, j=1}^{N} b_{i, j} x_{i} \partial_{x_{j}}-\partial_{t} .
\end{aligned}
$$


Since $\mathscr{L}_{s}$ satisfies assumptions [H.1-3], then (4.4) also applies to $u_{s}$. As a consequence,

$$
u\left(\delta_{s}(\bar{x}, \bar{t})\right)=u_{s}\left(\delta_{1-s_{0}}(\bar{x}, \bar{t})\right) \leq \widetilde{C} u_{s}(\bar{x}, \bar{t})=\widetilde{C} u\left(\delta_{s /\left(1-s_{0}\right)}(\bar{x}, \bar{t})\right) .
$$

Now let $n$ be the unique positive integer such that $\left(1-s_{0}\right)^{n+1} \leq s<\left(1-s_{0}\right)^{n}$. By applying $n$ times (4.5) we find

$$
u\left(\delta_{s}(\bar{x}, \bar{t})\right) \leq \widetilde{C}^{n} u\left(\delta_{r}(\bar{x}, \bar{t})\right), \quad r=s /\left(1-s_{0}\right)^{n} .
$$

On the other hand, the $\delta_{r}$-homogeneity of the norm $\|\cdot\|_{K}$ yields

$$
n=\frac{\ln \left\|\delta_{\left(1-s_{0}\right)^{n}}(\bar{x}, \bar{t})\right\|_{K}-\ln \|(\bar{x}, \bar{t})\|_{K}}{\ln \left(1-s_{0}\right)},
$$

so that

$$
\widetilde{C}^{n}=C_{1}\left\|\delta_{\left(1-s_{0}\right)^{n}}(\bar{x}, \bar{t})\right\|_{K}^{-\beta},
$$

with $C_{1}=\exp \left(-\frac{\ln \widetilde{C}}{\ln \left(1-s_{0}\right)} \ln \|(\bar{x}, \bar{t})\|_{K}\right)$, and $\beta=-\frac{\ln \widetilde{C}}{\ln \left(1-s_{0}\right)}>0$. Finally, since $s<\left(1-s_{0}\right)^{n}$ and $\beta>0$, (4.6) yields $\widetilde{C}^{n}<C_{1}\left\|\delta_{s}(\bar{x}, \bar{t})\right\|_{K}^{-\beta}$, so that

$$
u\left(\delta_{s}(\bar{x}, \bar{t})\right) \leq \frac{C_{1}}{\left\|\delta_{s}(\bar{x}, \bar{t})\right\|_{K}^{\beta}} u\left(\delta_{r}(\bar{x}, \bar{t})\right) .
$$

The proof is then accomplished by using (4.4).

We next show that if the boundary of $\Omega$ is a $\operatorname{Lip}_{K}$ surface, then it satisfies a strong Harnack connectivity condition.

Lemma 4.4. Let $Q_{r}^{\prime \prime}$ be as in (2.10) with $\left.\left.r \in\right] 0,1\right]$. Let $f: Q_{r}^{\prime \prime} \rightarrow \mathbb{R}$ be a $\operatorname{Lip}_{K}$ function such that $f(0,0)=0$. Then there exists a positive $\Lambda_{0}$, only depending on the operator $\mathscr{L}$ and on $M$, such that the following statement is true. For any $\Lambda \geq \Lambda_{0}$, let $x_{\Lambda}$ be as in (4.2), and set $z^{+}=\left(x_{\Lambda}, 1\right), z^{-}=\left(-x_{\Lambda}, 1\right)$. There exist $b \in] 0,1\left[\right.$, and two neighborhoods $U^{+}, U^{-}$of $x_{\Lambda}$ and $-x_{\Lambda}$ respectively, such that:

$$
\begin{aligned}
& \text { (i) } Z_{\delta_{b r} z^{+}, D_{b r} U^{+}}^{+}(x, t) \subseteq \Omega_{f, r}, \\
& \text { (ii) } Z_{\delta_{b r} z^{-}, D_{b r} U^{-}}(x, t) \cap \Omega_{f, r}=\emptyset,
\end{aligned}
$$

for every $(x, t) \in \Delta_{f, \frac{r}{2}}$. Here $U^{+}, U^{-}$and $b$ only depend on $\mathscr{L}, \Lambda$ and $M$.

Proof. We first prove (i) for $(x, t)=(0,0)$. In that order, we consider the cone

$$
K_{M, r}^{+}=\left\{(x, t) \in \operatorname{Int}\left(Q_{M, r}\right) \mid M\left\|\left(x^{\prime \prime}, t\right)\right\|_{K}^{\prime \prime}<x^{\prime}\right\},
$$

and we show that

$$
Z_{\delta_{\rho} z^{+}, D_{\rho} U^{+}}^{+}(0,0)=\left\{\delta_{s \rho}(x, 1) \mid x \in U^{+}, 0<s \leq 1\right\} \subseteq K_{M, r}^{+}, \quad \rho=b r .
$$


Here $\left.\Lambda \geq \Lambda_{0}(\mathscr{L}, M), b=b(\mathscr{L}, M, \Lambda) \in\right] 0,1\left[\right.$ and $U^{+}$is a neighborhood of $x_{\Lambda}$, depending on $\mathscr{L}, M$, and $\Lambda$. As a first step, we choose $\Lambda_{0}$ such that

$$
x_{\Lambda}^{\prime}>M\left\|\left(x_{\Lambda}^{\prime \prime}, 1\right)\right\|_{K}^{\prime \prime} \quad \text { for any } \Lambda>\Lambda_{0} .
$$

A direct computation shows that $\left(x_{\Lambda}\right)_{\prime \prime}^{(0)}=0$, and

$$
x_{\Lambda}^{(j)}=\frac{(-2)^{j} \Lambda}{(2 j+1) ! !}\left(B_{j}^{T} \cdots B_{1}^{T} e^{\prime}\right)^{(j)}, \quad j=1, \ldots, \kappa .
$$

Hence

$$
\left|x_{\Lambda}^{(j)}\right| \leq \Lambda C_{j}(B), \quad j=1, \ldots, \kappa,
$$

with the constants $C_{j}(B)$ 's have an obvious meaning. Hence, in order to prove (4.8), it is sufficient to prove the following inequality

$$
\Lambda>M\left(\sum_{j=1}^{\kappa}\left(\Lambda C_{j}(B)\right)^{\frac{1}{2 j+1}}+1\right) .
$$

However, this inequality which is trivially satisfied whenever $\Lambda$ is sufficiently large. Next, if we choose $b$ sufficiently small, then we have

$$
\delta_{\rho}\left(x_{\Lambda}, 1\right) \in K_{M, r}^{+}, \quad \rho=b r .
$$

As $K_{M, r}^{+}$is an open set, there exists a neighborhood $U^{+}$of $x_{\Lambda}$ such that $\left(D_{\rho} x, \rho^{2}\right) \in$ $K_{M, r}^{+}$for every $x \in U^{+}$. By the $\left(D_{r}^{\prime \prime}, r^{2}\right)$-homogeneity of the norm $\|\cdot\|_{K}^{\prime \prime}$, we also have $\delta_{s}\left(D_{\rho} x, \rho^{2}\right) \in K_{M, r}^{+}$for every $x \in U^{+}$and $\left.\left.s \in\right] 0,1\right]$. This proves (4.7) and then (i), recalling that $f$ is a $\operatorname{Lip}_{K}$ function, and the definition of $\Omega_{f, r}$.

We next prove (i) for every $(x, t) \in \Delta_{f, \frac{r}{2}}$. Let $\left.\varepsilon=\varepsilon(\mathscr{L}, M) \in\right] 0,1[$ be as in (2.11). From (4.7) it follows that

$$
Z_{\delta_{\varepsilon \rho} z^{+}, D_{\varepsilon \rho} U^{+}}^{+}(0,0) \subseteq K_{M, \varepsilon r}^{+}, \quad \rho=b r
$$

As a consequence, setting $K_{M, \varepsilon r}^{+}(x, t)=(x, t) \circ K_{M, \varepsilon r}^{+}$, we have

$$
Z_{\delta_{\varepsilon \rho} z^{+}, D_{\varepsilon \rho} U^{+}}^{+}(x, t) \subseteq K_{M, \varepsilon r}^{+}(x, t) \quad \text { for every } \quad(x, t) \in \mathbb{R}^{N+1} .
$$

We next show that

$$
K_{M, \varepsilon r}^{+}(x, t) \subseteq \Omega_{f, r}, \quad \text { for every }(x, t) \in \Delta_{f, \frac{r}{2}},
$$

and then (i) will directly follow from (4.11). Consider any $(\xi, \tau) \in K_{M, \varepsilon r}^{+}$. By (2.11), we have

$$
(x, t) \circ(\xi, \tau) \in Q_{M, r} \quad \text { for every }(x, t) \in \Delta_{f, \frac{r}{2}} \text {. }
$$


Hence, to prove (4.12) it is sufficient to show that

$$
f\left(\left(\xi+\exp \left(-\tau B^{T}\right) x\right)^{\prime \prime}, t+\tau\right)<\xi^{\prime}+x^{\prime},
$$

for every $(x, t) \in \Delta_{f, \frac{r}{2}}$. Note that, as $x^{\prime}=f\left(x^{\prime \prime}, t\right),(4.13)$ is equivalent to

$$
f\left(\left(\xi+\exp \left(-\tau B^{T}\right) x\right)^{\prime \prime}, t+\tau\right)-f\left(x^{\prime \prime}, t\right)<\xi^{\prime},
$$

which is trivially satisfied, since $f$ is $\operatorname{Lip}_{K}$, and $(\xi, \tau) \in K_{M, \varepsilon r}^{+}$. This proves (4.13) and then (4.12).

We next prove (ii). Consider the constant $b$ as in (4.10). Arguing as in the proof of (4.11), it is easy to see that

$$
Z_{\delta_{\varepsilon \rho} z^{-}, D_{\varepsilon \rho} U^{-}}^{-}(x, t) \subseteq K_{M, \varepsilon r}^{-}(x, t) \quad \text { for every } \quad(x, t) \in \mathbb{R}^{N+1} .
$$

Here $K_{M, \varepsilon r}^{-}(x, t)=(x, t) \circ K_{M, \varepsilon r}^{-}$and

$$
K_{M, \varepsilon r}^{-}=\left\{(x, t) \in \operatorname{Int}\left(Q_{M, \varepsilon r}\right) \mid x^{\prime}<-M\left\|\left(x^{\prime \prime}, t\right)\right\|_{K}^{\prime \prime}\right\} .
$$

Note that

$$
K_{M, \varepsilon r}^{-}(x, t) \cap \Omega_{f, r}=\emptyset, \quad \text { for every }(x, t) \in \Delta_{f, \frac{r}{2}},
$$

and hence the proof is complete.

Recall the definition of the ball $\mathcal{B}_{K}\left(z_{0}, r\right)$ in (2.5). As a plain consequence of Lemma 3.1 in [6] and of Lemma 4.4 -(ii), we have the following lemma.

Lemma 4.5. Let $Q_{r}^{\prime \prime}$ be as in (2.10) for $\left.\left.r \in\right] 0,1\right]$. Let $f: Q_{r}^{\prime \prime} \rightarrow \mathbb{R}$ be a $\operatorname{Lip}_{K}$ function such that $f(0,0)=0$. For every $\theta \in] 0,1\left[\right.$ there exists $\left.\left.\rho_{\theta} \in\right] 0,1\right]$ such that

$$
\sup _{\Omega_{f, r} \cap \mathcal{B}_{K}\left(z_{0}, s \rho_{\theta}\right)} u \leq \theta \sup _{\Omega_{f, r} \cap \mathcal{B}_{K}\left(z_{0}, s\right)} u
$$

for every non-negative solution $u$ to $\mathscr{L} u=0$ in $\Omega_{f, r}$ such that $u=0$ on $\Delta_{f, \frac{r}{2}}$, and for every $z_{0} \in \Delta_{f, \frac{r}{2}}$ and $s>0$ such that $\mathcal{B}_{K}\left(z_{0}, s\right) \cap \partial \Omega_{f, r} \subset \Delta_{f, \frac{r}{2}}$.

We end this section by proving the following lemma.

Lemma 4.6. Let $Q_{r}^{\prime \prime}$ be as in (2.10) with $\left.\left.r \in\right] 0,1\right]$. Let $f: Q_{r}^{\prime \prime} \rightarrow \mathbb{R}$ be a $\operatorname{Lip}_{K}$ function such that $f(0,0)=0$. Then there exist three constants $C_{2}>1, \Lambda_{1} \geq \Lambda_{0}$ and $\left.\left.\rho_{0} \in\right] 0,1\right]$ only depending on the operator $\mathscr{L}$ and on $M$, such that $C_{2} \rho_{0}<1$ and the following statement is true. If $z^{+}=\left(x_{\Lambda}, 1\right)$ is as in Lemma 4.4 for some $\Lambda \geq \Lambda_{1}$, then, for every $\left.\left.\rho \in\right] 0, \rho_{0}\right]$ and every $(\xi, \tau) \in \Omega_{f, r \rho}$, there exist $(x, t) \in$ $\Delta_{f, C_{2} r \rho}$ and $\left.\widetilde{s} \in\right] 0, r \rho[$ such that

$$
(\xi, \tau)=(x, t) \circ \delta_{\widetilde{s}} z^{+} .
$$


Proof. Let $\left.\left.\rho_{0} \in\right] 0,1\right]$ be a positive constant that will be chosen later. Consider the path $\gamma(s)=(\xi, \tau) \circ \delta_{s}\left(\left(x_{\Lambda}, 1\right)^{-1}\right)$ for $s>0$, where $(\xi, \tau)$ is any point of $\Omega_{f, r \rho_{0}}$. By a direct computation we find

$$
\left(x_{\Lambda}, 1\right)^{-1}=\left(-\Lambda e^{\prime},-\frac{\Lambda}{3} B_{1}^{T} e^{\prime}, \ldots,-\left(\sum_{i=0}^{\kappa} \frac{(-2)^{\kappa-i} \Lambda}{i !(2 \kappa-2 i+1) ! !}\right) B_{\kappa}^{T} \cdots B_{1}^{T} e^{\prime},-1\right),
$$

so that

$$
\begin{aligned}
\gamma(s)=( & -s \Lambda e^{\prime}+\xi^{(0)},-s^{3} \frac{\Lambda}{3} B_{1}^{T} e^{\prime}+s^{2} B_{1}^{T} \xi^{(0)}+\xi^{(1)}, \ldots, \\
& -s^{2 \kappa+1} \sum_{i=0}^{\kappa} \frac{(-2)^{\kappa-i} \Lambda}{i !(2 \kappa-2 i+1) ! !} B_{\kappa}^{T} \cdots B_{1}^{T} e^{\prime} \\
& \left.+\sum_{i=1}^{\kappa} \frac{s^{2 i}}{i !} B_{\kappa}^{T} \cdots B_{\kappa-i+1}^{T} \xi^{(\kappa-i)}+\xi^{(\kappa)}, \tau-s^{2}\right),
\end{aligned}
$$

for any $\Lambda \geq \Lambda_{0}$. We claim that it is possible to determine a $\left.\left.\rho_{0}=\rho_{0}(\mathscr{L}, M) \in\right] 0,1\right]$ and to choose $\Lambda_{1}=\Lambda_{1}(\mathscr{L}, M) \geq \Lambda_{0}$, in order to have

$$
\left.\left.\gamma(s) \in Q_{M, r} \quad \text { for every } s \in\right] 0, r \rho_{0}\right], \quad \text { and } \gamma\left(r \rho_{0}\right) \notin \Omega_{f, r},
$$

for any $\Lambda \geq \Lambda_{1}$. We next choose $\left.\left.\rho_{0}=\rho_{0}(\mathscr{L}, M) \in\right] 0,1\right]$ satisfying the first statement of (4.16). With this aim, it is sufficient to show that

$$
\left|-s \Lambda+\xi^{\prime}\right| \leq 4 M r, \quad\left|\tau-s^{2}\right| \leq 2 r^{2}, \quad\left|\gamma_{i}(s)\right| \leq r^{\alpha_{i}}, \quad i=m+1, \ldots, N,
$$

for every $\left.s \in] 0, r \rho_{0}\right]$. Since $(\xi, \tau) \in Q_{M, r \rho_{0}}$, we have

$$
\left|-s \Lambda+\xi^{\prime}\right| \leq r \rho_{0}(\Lambda+4 M), \quad\left|\tau-s^{2}\right| \leq 3\left(r \rho_{0}\right)^{2}
$$

for every $\left.s \in] 0, r \rho_{0}\right]$. Moreover,

$$
\begin{aligned}
\left|\gamma^{(j)}(s)\right| & \leq \Lambda c_{j+1} s^{2 j+1}+\sum_{l=1}^{j} \widetilde{c}_{l} s^{2 l}\left|\xi^{(j-l)}\right|+\left|\xi^{(j)}\right| \\
& \leq\left(r \rho_{0}\right)^{2 j+1}\left(\Lambda c_{j+1}+\sum_{l=1}^{j} c_{l}+m_{j}\right)
\end{aligned}
$$

for any $j=1, \ldots, \kappa$, and for every $\left.s \in] 0, r \rho_{0}\right]$. Here the $c_{l}$ 's are positive constants only dependent on $M$ and on the matrix $B$. Hence, condition (4.17) is satisfied by choosing

$$
\rho_{0}<C_{2}^{-1} \text {, }
$$


where

$$
C_{2}:=\max \left\{1+\frac{\Lambda}{4 M}, \sqrt{\frac{3}{2}}, \max _{j=1, \ldots, \kappa}\left(\Lambda c_{j+1}+\sum_{l=1}^{j} c_{l}+m_{j}\right)^{\frac{1}{2 j+1}}\right\} .
$$

This proves that

$$
\left.\left.\gamma(s) \in Q_{M, C_{2} r \rho_{0}} \text { for every } s \in\right] 0, r \rho_{0}\right] .
$$

Note that $C_{2} \rho_{0}<1$ yields $Q_{M, C_{2} r \rho_{0}} \subset Q_{M, r}$, hence the first statement of (4.16) is proved.

It remains to prove the last part of (4.16). We will show that it is possible to choose of $\Lambda_{1}$ such that

$$
\gamma\left(r \rho_{0}\right)^{\prime}<f\left(\gamma\left(r \rho_{0}\right)^{\prime \prime}, \tau-\left(r \rho_{0}\right)^{2}\right)
$$

for every $\Lambda \geq \Lambda_{1}$. To this aim, as $f$ is $\operatorname{Lip}_{K}$, it is enough to show that

$$
M\left\|\left(\gamma\left(r \rho_{0}\right)^{\prime \prime}, \tau-\left(r \rho_{0}\right)^{2}\right)\right\|_{K}^{\prime \prime}<r \rho_{0} \Lambda-\xi^{\prime} .
$$

As in the first estimate of (4.18) we have

$$
r \rho_{0} \Lambda-\xi^{\prime} \geq r \rho_{0}(\Lambda-4 M),
$$

and since we want the right handside in (4.22) to be positive we require that $\Lambda_{1}>$ $4 M$. From (4.18) and (4.19), it follows that

$$
\left\|\left(\gamma\left(r \rho_{0}\right)^{\prime \prime}, \tau-\left(r \rho_{0}\right)^{2}\right)\right\|_{K}^{\prime \prime} \leq r \rho_{0}\left(m-1+\sum_{j=1}^{\kappa}\left(\Lambda c_{j+1}+\sum_{l=1}^{j} c_{l}+m_{j}\right)^{\frac{1}{2 j+1}}+\sqrt{3}\right) .
$$

Now, choosing $\Lambda_{1}$ so large that

$$
\Lambda_{1}>M\left(m+3+\sum_{j=1}^{\kappa}\left(\Lambda_{1} c_{j+1}+\sum_{l=1}^{j} c_{l}+m_{j}\right)^{\frac{1}{2 j+1}}+\sqrt{3}\right),
$$

we see that (4.22) follows from (4.23) and (4.24). This completes the proof of (4.16).

From the second statement of (4.16), and from (4.21), it follows that we can find $\widetilde{s} \in] 0, r \rho_{0}[$ such that

$$
\gamma(\widetilde{s})=:(x, t) \in \Delta_{f, C_{2} r \rho_{0}},
$$

and the proof of the lemma is accomplished. Indeed, by (4.20), the result holds true also for every $\left.\rho \in] 0, \rho_{0}\right]$. 


\section{Proof of the main result}

Proof of Proposition 2.2. As in (2.7), we set $x^{\prime}=x_{j}$, for some $j=1, \ldots, m$. Consider $z \in \Sigma$ and $r>0$ such that

$$
\Sigma \cap C_{r}(z)=\left\{(\xi, \tau) \in C_{r}(z) \mid f\left(\xi^{\prime \prime}, \tau\right)=\xi^{\prime}\right\},
$$

where $f$ is $\operatorname{Lip}(1,1 / 2)$. Since $C_{r}(z)$ is an open set, there exist some positive $M_{z}$ and $r_{z}$ satisfying $Q_{M_{z}, 2 r_{z}}(z) \subseteq C_{r}(z)$. To prove Proposition 2.2 it is enough to show that $\left.f\right|_{Q_{2 r_{z}}^{\prime \prime}}$ is a $\operatorname{Lip}_{K}$ function. To accomplish this we let $\left(y^{\prime \prime}, s\right),\left(\xi^{\prime \prime}, \tau\right) \in Q_{2 r_{z}}^{\prime \prime}$ and we prove that there exists a positive constant $C=C\left(Q_{2 r_{z}}^{\prime \prime}\right)$ satisfying

$$
\left|y^{\prime \prime}-\xi^{\prime \prime}\right|+|s-\tau|^{\frac{1}{2}} \leq C\left\|\left(\left(y-\exp \left((\tau-s) B^{T}\right) \xi\right)^{\prime \prime}, s-\tau\right)\right\|_{K}^{\prime \prime} .
$$

Recall the notation in (2.2) and (2.7) and note that

$$
\left(y-\exp \left((\tau-s) B^{T}\right) \xi\right)_{\prime \prime}^{(0)}=y_{\prime \prime}^{(0)}-\xi_{\prime \prime}^{(0)} .
$$

Moreover,

$$
\begin{aligned}
\left|y^{(1)}-\xi^{(1)}\right| & \leq\left|y^{(1)}-\xi^{(1)}-(\tau-s) B_{1}^{T} \xi^{(0)}\right|+|s-\tau|\left|B_{1}^{T} \xi^{(0)}\right| \\
& \leq C_{1,1}^{\frac{2}{3}}\left|y^{(1)}-\xi^{(1)}-(\tau-s) B_{1}^{T} \xi^{(0)}\right|^{\frac{1}{3}}+C_{1,2}|s-\tau|^{\frac{1}{2}},
\end{aligned}
$$

for two positive constants $C_{1,1}$ and $C_{2,2}$, depending only on the set $Q_{2 r_{z}}^{\prime \prime}$. Analogously, for any $l=2, \ldots, \kappa$, there exist $C_{l, i}=C_{l, i}\left(Q_{2 r_{z}}^{\prime \prime}\right)>0, i=1,2$, such that

$$
\begin{aligned}
\left|y^{(l)}-\xi^{(l)}\right| \leq\left|y^{(l)}-\xi^{(l)}-\sum_{i=1}^{l} \frac{(\tau-s)^{i}}{i !} B_{l}^{T} \cdots B_{l-i+1}^{T} \xi^{(l-i)}\right| \\
\quad+|s-\tau|\left|B_{l}^{T} \xi^{(l-1)}+\sum_{i=2}^{l} \frac{(\tau-s)^{i-1}}{i !} B_{l}^{T} \cdots B_{l-i+1}^{T} \xi^{(l-i)}\right| \\
\leq C_{l, 1}^{\frac{2 l}{2 l+1}}\left|y^{(l)}-\xi^{(l)}-\sum_{i=1}^{l} \frac{(\tau-s)^{i}}{i !} B_{l}^{T} \cdots B_{l-i+1}^{T} \xi^{(l-i)}\right|^{\frac{1}{2 l+1}} \\
+C_{l, 2}|s-\tau|^{\frac{1}{2}} .
\end{aligned}
$$

Recalling (2.8) we see that this proves (5.1).

Proof of Proposition 2.3. It is not restrictive to assume $\left(x_{0}, t_{0}\right)=(0,0)$ and $r=$ 1. Let $(\widetilde{x}, \widetilde{t})=\delta_{b} z^{+}$and $U=D_{b} U^{+}$, where $b, z^{+}$and $U^{+}$are as in Lemma 4.4. Consider any $\widetilde{s} \in] 0,1\left[\right.$. By Lemma 4.2 , the point $\delta_{\widetilde{s}}(\widetilde{x}, \widetilde{t})$ belongs to an $\mathscr{L}$-admissible path starting from $(\widetilde{x}, \widetilde{t})$. Actually, the same argument used at the 
beginning of the proof of Proposition 3.2 shows that $\delta_{\widetilde{s}}(\tilde{x}, \widetilde{t})$ is an interior point of $\mathscr{A}_{(\widetilde{x}, \widetilde{t})}\left(Z_{\widetilde{x}, \tilde{t}, U}^{+}(0,0)\right)$. Hence there exists a positive $\widetilde{\rho}$ such that

$$
\widetilde{K}:=\overline{\mathcal{B}_{K}\left(\delta_{\widetilde{s}}(\widetilde{x}, \widetilde{t}), \widetilde{\rho}\right)} \subset \operatorname{Int}\left(\mathscr{A}_{(\widetilde{x}, \tilde{t})}\left(Z_{\widetilde{x}, \tilde{t}, U}^{+}(0,0)\right)\right) .
$$

By Theorem 3.1 there exists a positive constant $C_{\widetilde{K}}$ such that $\sup _{\widetilde{K}} u \leq C_{\widetilde{K}} u(\widetilde{x}, \widetilde{t})$. By the linearity of $\mathscr{L}$, it is not restrictive to assume $C_{\widetilde{K}} u(\widetilde{x}, \widetilde{t})=1$, and hence that

$$
\sup _{\widetilde{K}} u \leq 1 .
$$

Moreover, by the continuity of the function $(\xi, \tau) \mapsto(\xi, \tau) \circ \delta_{\widetilde{S}}(\widetilde{x}, \widetilde{t})$, there exists $\left.\varepsilon_{1} \in\right] 0,1[$ such that

$$
(\xi, \tau) \circ \delta_{\widetilde{s}}(\widetilde{x}, \widetilde{t}) \in \widetilde{K} \quad \text { for every } \quad(\xi, \tau) \in \Delta_{f, \varepsilon_{1}} .
$$

We next follow a classical argument also used in our previous work [6]. We fix a suitably large constant $\lambda$ and we assume, by contradiction, that there exists $z_{1} \in$ $\Omega_{f, c}$ satisfying $u\left(z_{1}\right)>\lambda$. To this aim, we choose $\left.\theta \in\right] 0, \mathbf{c}^{-\beta}[$, where $\mathbf{c}$ is the constant in (2.3), and $\beta$ is as in Lemma 4.3. Then we set

$$
\begin{aligned}
\varepsilon_{0} & <\min \left\{\rho_{0}, \varepsilon_{1}, b \widetilde{s}, \frac{c_{M}^{\prime}}{4 c_{M}^{\prime \prime} \mathbf{c}}\right\}, \quad \sigma<\min \left\{1, \frac{c_{M}^{\prime}}{2 C \mathbf{c}}, \frac{c_{M}^{\prime \prime}}{C_{2}}\right\}, \\
\lambda & >\max \left\{1, C_{1}\left(\frac{4 \mathbf{c}}{\rho_{\theta} c_{M}^{\prime}}\right)^{\beta}, \frac{C_{1}}{\theta}\left(\frac{\mathbf{c}\left(\rho_{\theta}^{-1}+\mathbf{c}\right) 2 C_{2}}{c_{M}^{\prime} \varepsilon_{0}} \sum_{j=1}^{\infty}\left(\mathbf{c} \theta^{\frac{1}{\beta}}\right)^{j}\right)^{\beta}\right\},
\end{aligned}
$$

where $c_{M}^{\prime}, c_{M}^{\prime \prime}$ are the constants in (2.12), $C_{1}$ is as in Lemma 4.3, $b$ is as in Lemma 4.4, $\left.\left.\rho_{\theta} \in\right] 0,1\right]$ is as in Lemma 4.5, $\rho_{0}$ and $C_{2}$ are as in Lemma 4.6, and $\varepsilon_{1}$ is as in (5.4). Note that the series in (5.5) is convergent since $\theta \in] 0, \mathbf{c}^{-\beta}[$.

Let

$$
c=\frac{\sigma \varepsilon_{0}}{c_{M}^{\prime \prime}}
$$

and let $z_{1} \in \Omega_{f, c}$ be such that $u\left(z_{1}\right)>\lambda$. Note that (2.12) and the choice of $\sigma$ in (5.5) imply that

$$
z_{1} \in \mathcal{B}_{K}\left(0, \sigma \varepsilon_{0}\right) \cap \Omega_{f, \frac{\varepsilon_{0}}{C_{2}}} .
$$

We next show that there exists a sequence $\left\{z_{j}\right\}_{j}$ such that

$$
z_{j} \in \Omega_{f, \frac{\varepsilon_{0}}{C_{2}},}, \quad u\left(z_{j}\right)>\lambda \theta^{1-j},
$$

for every $j \in \mathbb{N}$. We will see that the properties of $z_{j}$ in (5.8) enable us to determine a point $w_{j} \in \Delta_{f, \varepsilon_{0}}$ and a $\left.s_{j} \in\right] 0,1[$ satisfying

$$
z_{j}=w_{j} \circ \delta_{s_{j}}\left(\delta_{\widetilde{s}}(\widetilde{x}, \widetilde{t})\right), \quad \rho_{j}:=d_{K}\left(z_{j}, w_{j}\right) \leq C_{1}^{\frac{1}{\beta}} \lambda^{-\frac{1}{\beta}} \theta^{\frac{j-1}{\beta}},
$$


for every $j \in \mathbb{N}$. As a consequence of the inequality in (5.9), we get $d_{K}\left(z_{j}, \Delta_{f, \varepsilon_{0}}\right) \leq$ $d_{K}\left(z_{j}, w_{j}\right) \rightarrow 0$ as $j \rightarrow \infty$, then $u\left(z_{j}\right)$ vanishes as $j \rightarrow \infty$. This will then contradict the inequality in (5.8) and hence

$$
\sup _{\Omega_{f, c}} u \leq \lambda C_{\widetilde{K}} u(\widetilde{x}, \widetilde{t}) .
$$

In particular, the proof will be accomplished.

We next prove (5.8) by induction. Our choice of $z_{1}$ proves the claim for $j=1$. Assume that (5.8) is satisfied for $j=k$. By Lemma 4.6, as $\varepsilon_{0} C_{2}^{-1}<\rho_{0}$, there exist

$$
\left.w_{k} \in \Delta_{f, \varepsilon_{0}}, \quad s_{k} \in\right] 0, \frac{\varepsilon_{0}}{C_{2} b \widetilde{s}}[,
$$

such that $z_{k}=w_{k} \circ \delta_{s_{k}}\left(\delta_{\widetilde{s}}(\tilde{x}, \widetilde{t})\right)$. Note that the choice of $\varepsilon_{0}$ in (5.5) implies that $\left.s_{k} \in\right] 0,1\left[\right.$, and that $w_{k} \circ \delta_{\widetilde{s}}(\widetilde{x}, \widetilde{t}) \in \widetilde{K}$, by (5.4). From Lemma 4.3 it follows that

$$
\lambda \theta^{1-k}<u\left(z_{k}\right)=u\left(w_{k} \circ \delta_{s_{k}}\left(\delta_{\widetilde{s}}(\tilde{x}, \tilde{t})\right)\right) \leq \frac{C_{1}}{s_{k}^{\beta} \widetilde{s}^{\beta} \|\left(\widetilde{x}, \widetilde{t} \|_{K}^{\beta}\right.} u\left(w_{k} \circ \delta_{\widetilde{s}}(\tilde{x}, \widetilde{t})\right) .
$$

Hence, (5.3) gives

$$
\rho_{k}:=d_{K}\left(z_{k}, w_{k}\right) \leq C_{1}^{\frac{1}{\beta}} \lambda^{-\frac{1}{\beta}} \theta^{\frac{k-1}{\beta}} .
$$

We next claim that

$$
\mathcal{B}_{K}\left(w_{k}, \rho_{\theta}^{-1} \rho_{k}\right) \subseteq Q_{M, \frac{1}{2}}, \quad \text { for every } k \in \mathbb{N} .
$$

Indeed, consider any $z \in \mathcal{B}_{K}\left(w_{k}, \rho_{\theta}^{-1} \rho_{k}\right)$. By using (2.3), (5.11), and recalling that $\Delta_{f, \varepsilon_{0}} \subseteq \mathcal{B}_{K}\left(0, c_{M}^{\prime \prime} \varepsilon_{0}\right)$ by (2.12), we have

$$
\begin{aligned}
\|z\|_{K} & \leq \mathbf{c}\left(\left\|w_{k}\right\|_{K}+\left\|w_{k}^{-1} \circ z\right\|_{K}\right) \leq \mathbf{c}\left(c_{M}^{\prime \prime} \varepsilon_{0}+\rho_{\theta}^{-1} \rho_{k}\right) \\
& \leq \mathbf{c}\left(c_{M}^{\prime \prime} \varepsilon_{0}+\rho_{\theta}^{-1} C_{1}^{\frac{1}{\beta}} \lambda^{-\frac{1}{\beta}}\right)<\frac{c_{M}^{\prime}}{2},
\end{aligned}
$$

thanks to the bounds of $\varepsilon_{0}$ and $\lambda$ in (5.5). This inequality, together with (2.12), proves (5.12). As a consequence, we have

$$
\mathcal{B}_{K}\left(w_{k}, \rho_{\theta}^{-1} \rho_{k}\right) \cap \partial \Omega_{f, 1} \subset \Delta_{f, \frac{1}{2}}, \quad \text { for every } k \in \mathbb{N} .
$$

Then, by Lemma 4.5 ,

$$
\lambda \theta^{1-k}<u\left(z_{k}\right) \leq \sup _{\Omega_{f, 1} \cap \mathcal{B}_{K}\left(w_{k}, \rho_{k}\right)} u \leq \theta \sup _{\Omega_{f, 1} \cap \mathcal{B}_{K}\left(w_{k}, \rho_{\theta}^{-1} \rho_{k}\right)} u .
$$


Hence, there exists $z_{k+1} \in \overline{\Omega_{f, 1} \cap \mathcal{B}_{K}\left(w_{k}, \rho_{\theta}^{-1} \rho_{k}\right)}$ such that

$$
u\left(z_{k+1}\right)>\lambda \theta^{-k}
$$

This shows the second statement of (5.8) for $j=k+1$. Aiming to conclude the proof, it is enough to check that $z_{k+1} \in \Omega_{f, \frac{\varepsilon_{0}}{C_{2}}}$. By repeatedly using the pseudotriangular inequality (2.3), we get

$$
\begin{aligned}
\left\|z_{k+1}\right\|_{K} & \leq \mathbf{c}\left(\left\|z_{1}\right\|_{K}+\left\|z_{1}^{-1} \circ z_{k+1}\right\|_{K}\right) \\
& \leq \mathbf{c}\left(\left\|z_{1}\right\|_{K}+\sum_{j=1}^{k} \mathbf{c}^{j}\left\|z_{j}^{-1} \circ z_{j+1}\right\|_{K}\right) .
\end{aligned}
$$

We also have

$$
\begin{aligned}
\left\|z_{j}^{-1} \circ z_{j+1}\right\|_{K} & \leq \mathbf{c}\left(\left\|z_{j}^{-1} \circ w_{j}\right\|_{K}+\left\|w_{j}^{-1} \circ z_{j+1}\right\|_{K}\right) \\
& \leq \mathbf{c}\left(\mathbf{c} \rho_{j}+\rho_{\theta}^{-1} \rho_{j}\right)<\mathbf{c}\left(\mathbf{c}+\rho_{\theta}^{-1}\right) C_{1}^{\frac{1}{\beta}} \lambda^{-\frac{1}{\beta}} \theta^{\frac{j-1}{\beta}},
\end{aligned}
$$

by (5.11). By using the above inequality in (5.13), and recalling (5.7), we find

$$
\left\|z_{k+1}\right\|_{K} \leq \mathbf{c}\left(\sigma \varepsilon_{0}+\mathbf{c}\left(\mathbf{c}+\rho_{\theta}^{-1}\right) C_{1}^{\frac{1}{\beta}}(\lambda \theta)^{-\frac{1}{\beta}} \sum_{j=1}^{\infty}\left(\theta^{\frac{1}{\beta}} \mathbf{c}\right)^{j}\right)<\frac{c_{M}^{\prime} \varepsilon_{0}}{C_{2}}
$$

because of our choice of $\sigma$ and $\lambda$ in (5.5). This proves that

$$
z_{k+1} \in \mathcal{B}_{K}\left(0, \frac{c_{M}^{\prime} \varepsilon_{0}}{C_{2}}\right) \cap \Omega_{f, 1} \subseteq \Omega_{f, \frac{\varepsilon_{0}}{c_{2}}}
$$

by (2.12). The proof is accomplished.

Proof of Theorem 1.1. Consider any $z_{j}$, for $j=1, \ldots, k$, and apply Proposition 2.3 to $Q_{M_{j}, 2 r_{j}}\left(z_{j}\right)$. We only need to show that the constants $c$ and $C$ do not depend on the coordinate system relevant to $e_{j}^{\prime}$. We rely on the following elementary statement. For every $M>0$ there exists $\varepsilon>0$ such that, if $\Delta_{f, 2 r}$ is a $\operatorname{Lip}_{K}$ surface with Lipschitz constant $M$ and with respect to a $e^{\prime} \in \mathbb{R}^{m}$, then $\Delta_{f, r}$ is a Lip $\operatorname{lirface}_{K}$ with Lipschitz constant $2 M$ and with respect to $\widetilde{e}^{\prime}$, for every $\widetilde{e}^{\prime} \in \mathbb{R}^{m}$ such that $\left\|\widetilde{e}^{\prime}\right\|=1$ and $\left\|\widetilde{e}^{\prime}-e^{\prime}\right\| \leq \varepsilon$. By compactness, there exists a finite set $\widetilde{e}_{1}^{\prime}, \ldots, \widetilde{e}_{n}^{\prime}$ of unit vectors of $\mathbb{R}^{m}$ such that $B\left(\widetilde{e}_{1}^{\prime}, \varepsilon\right), \ldots, B\left(\widetilde{e}_{n}^{\prime}, \varepsilon\right)$ cover the unit sphere of $\mathbb{R}^{m}$. Hence there exists $\widetilde{e}_{i}^{\prime}$, with $i \in\{1, \ldots, n\}$, such that $\Delta_{f_{j}, r_{j}}\left(z_{j}\right)$ is a Lip $\operatorname{Lirface}_{K}$ with respect to $\widetilde{e}_{i}^{\prime}$ and with Lipschitz constant $2 M_{j}$. To conclude the proof of Theorem 1.1 it is sufficient to set $c=\min \left\{c_{1}, \ldots, c_{n}\right\}$ and $C=\max \left\{C_{1}, \ldots, C_{n}\right\}$, where $c_{i}$ and $C_{i}$ are the constants relevant to $\operatorname{Lip}_{K}$ functions of Lipschitz constant $2 M$, with respect to $\widetilde{e}_{i}^{\prime}, i=1, \ldots, n$. 
Proof of Proposition 2.4. As in the proof of Proposition 2.3, it is not restrictive to assume $\left(x_{0}, t_{0}\right)=(0,0)$ and $r=1$. Consider the point $\widetilde{z}=(0, \widetilde{t})$ for $\widetilde{t}$ positive and to be chosen. Arguing as in the proof of Lemma 4.4, it is easy to see that we can choose a sufficiently small positive $b$, and a suitable neighborhood of the origin $\widetilde{U}$, such that

$$
\begin{aligned}
& Z_{\delta_{b} \widetilde{z}, \widetilde{U}}^{+}(x, t) \subset \widetilde{K}_{M, 1}^{+}(x, t) \\
& :=\left\{(x, t) \circ(\xi, \tau) \mid(\xi, \tau) \in Q_{1,1, M}, \tau>M\left(\left|\xi^{(1)}\right|^{\frac{2}{3}}+\cdots+\left|\xi^{(\kappa)}\right|^{\frac{2}{2 \kappa+1}}\right)\right\},
\end{aligned}
$$

for every $(x, t) \in \mathbb{R}^{N+1}$. By the continuity of the operation "o" and by the Lipschitz continuity of $g$, it is possible to find a suitably small $\varepsilon$ such that

$$
\widetilde{K}_{M, \varepsilon}^{+}(x, t) \subset \widetilde{\Omega}_{g, 1} \quad \text { for every }(x, t) \in \widetilde{\Delta}_{g, 1 / 2} .
$$

This proves that $Z_{\delta_{b}, \tilde{z}, \widetilde{U}}^{+}(x, t) \subset \widetilde{\Omega}_{g, 1}$, for every $(x, t) \in \widetilde{\Delta}_{g, 1 / 2}$. Analogously, we can show that $Z_{\delta_{b}}^{-} \widetilde{z}, \widetilde{U}(x, t) \cap \widetilde{\Omega}_{g, 1}=\emptyset$, for every $(x, t) \in \widetilde{\Delta}_{g, 1 / 2}$. Then a conclusion similar to Lemma 4.4 and Lemma 4.5 plainly follows. To prove an analogous statement of Lemma 4.6 is even simpler. Indeed, since we in this case have $\widetilde{z}=(0, \widetilde{t})$ we can simply consider the path

$$
\gamma(s)=(\xi, \tau) \circ\left(0,-s^{2} \widetilde{t}\right)=\left(\exp \left(s^{2} \widetilde{t} B^{T}\right) \xi, \tau-s^{2} \widetilde{t}\right), \quad s>0 .
$$

Arguing as in the proof of Lemma 4.6, it easy to find $\left.\left.\rho_{0} \overline{\bar{\Omega}} \rho_{0}(\mathscr{L}, M) \in\right] 0,1\right]$ such that $\gamma(s) \in Q_{1,1, M}$ for every $\left.\left.s \in\right] 0, \rho_{0}\right]$ and $\gamma\left(\rho_{0}\right) \notin \widetilde{\Omega}_{g, 1}$. We omit further details.

Proof of Proposition 2.5. We first explain the reason we choose a small Lipschitz constant $M$. We aim to find a cone $Z_{\delta_{r}}^{+} \widetilde{z}, D_{r} \widetilde{U}\left(x_{0}, t_{0}\right)$ contained in $\widetilde{\Omega}_{g}$, in order to rely on the argument used in Proposition 2.4. To show that $\left(x_{0}, t_{0}\right) \circ \delta_{r} \widetilde{z} \in \widetilde{\Omega}_{g}$, it is sufficient to have

$$
t>M\left|\exp \left(t B^{T}\right) x_{0}-x_{0}\right| \geq\left|g\left(\exp \left(t B^{T}\right) x_{0}\right)-g\left(x_{0}\right)\right|, \quad 0<t \leq M c_{Q},
$$

by the Lipschitz continuity of $g$. Since $\exp \left(t B^{T}\right) x_{0}=x_{0}+t B^{T} x_{0}+o(t)$ as $t \rightarrow 0$, the above requirement is satisfied for any positive $t$ small enough, provided that the Lipschitz constant $M$ satisfies $M \sup _{x \in Q}\left\|B^{T} x\right\|<1$. Once this condition is satisfied, we build a cone $Z_{\delta_{r}}^{+} \widetilde{z}, D_{r} \widetilde{U}\left(x_{0}, t_{0}\right)$ contained in $\widetilde{\Omega}_{g}$ and we conclude the proof of Proposition 2.5 exactly as the one of Proposition 2.4. We omit further details.

\section{References}

[1] P. Bauman, Positive solutions of elliptic equations in nondivergence form and their adjoints, Ark. Mat. 22 (1984), 153-173. 
[2] L. Caffarelli, E. Fabes, S. Mortola and S. Salsa, Boundary behavior of nonnegative solutions of elliptic operators in divergence form, Indiana Univ. Math. J. 30 (1981), 621-640.

[3] L. CARLESON, On the existence of boundary values for harmonic functions in several variables, Ark. Mat. 4 (1962), 393-399.

[4] L. CAPOGNA and N. GAROFALO, Boundary behavior of nonnegative solutions of subelliptic equations in NTA domains for Carnot-Carathéodory metrics, J. Fourier Anal. Appl. 4 (1998), 403-432.

[5] L. Capogna, N. Garofalo and D. M. Nhieu, Examples of uniform and NTA domains in Carnot groups, In: "Proceedings on Analysis and Geometry" (Russian) (Novosibirsk Akademgorodok, 1999), 103-121, Izdat. Ross. Akad. Nauk Sib. Otd. Inst. Mat., Novosibirsk, 2000.

[6] C. Cinti, K. Nyström and S. Polidoro, A boundary estimate for non-negative solutions to Kolmogorov operators in non-divergence form, Ann. Mat. Pura Appl. (4) 191 (2012), 1-23.

[7] C. Cinti, K. Nyström and S. Polidoro, A note on Harnack inequalities and propagation sets for a class of hypoelliptic operators, Potential Anal. 33 (2010), 341-354.

[8] D. Danielli, N. Garofalo and A. Petrosyan, The sub-elliptic obstacle problem: $C^{1, \alpha}$ regularity of the free boundary in Carnot groups of step two, Adv. Math. 211 (2007), 485-516.

[9] D. Danielli, N. Garofalo and S. SAlsa, Variational inequalities with lack of ellipticity. I. Optimal interior regularity and non-degeneracy of the free boundary, Indiana Univ. Math. J. 52 (2003), 361-398.

[10] M. Di Francesco, A. PascuCCI and S. Polidoro, The obstacle problem for a class of hypoelliptic ultraparabolic equations, Proc. R. Soc. Lond. Ser. A Math. Phys. Eng. Sci. 464 (2008), 155-176.

[11] M. Di FrANCESCO and S. POLIDORO, Schauder estimates, Harnack inequality and Gaussian lower bound for Kolmogorov-type operators in non-divergence form, Adv. Differential Equations 11 (2006), 1261-1320.

[12] E. Fabes, N. Garofalo, S. Marín-Malave and S. Salsa, Fatou theorems for some nonlinear elliptic equations, Rev. Mat. Iberoamericana 4 (1988), 227-251.

[13] E. B. Fabes, N. Garofalo and S. SAlSA, A backward Harnack inequality and Fatou theorem for nonnegative solutions of parabolic equations, Illinois J. Math. 30 (1986), 536565 .

[14] E. B. FABES andC. E. KeNIG, Examples of singular parabolic measures and singular transition probability densities, Duke Math. J. 48 (1981), 845-856.

[15] E. B. FABES AND M. V. SAFONOV, Behavior near the boundary of positive solutions of second order parabolic equations, In: "Proceedings of the conference dedicated to Professor Miguel de Guzmán" (El Escorial, 1996), Vol. 3 (1997), 871-882.

[16] E. B. FABES, M. V. SAFONOV and Y. YUAN, Behavior near the boundary of positive solutions of second order parabolic equations. II, Trans. Amer. Math. Soc. 351 (1999), 4947-4961.

[17] E. B. FABES and D. W. STROOCK, A new proof of Moser's parabolic Harnack inequality using the old ideas of Nash, Arch. Ration. Mech. Anal. 96 (1986), 327-338.

[18] F. FERRARI and B. FRANCHI, Geometry of the boundary and doubling property of the harmonic measure for Grushin type operators, Rend. Sem. Mat. Univ. Politec. Torino 58 (2000), 281-299 (2002). Partial differential operators (Torino, 2000).

[19] F. FERRARI and B. FRANCHI, A local doubling formula for the harmonic measure associated with subelliptic operators and applications, Comm. Partial Differential Equations 28 (2003), 1-60.

[20] M. Frentz, N. Garofalo, E. Götmark, I. Munive and K. Nyström, Nondivergence form parabolic equations associated with non-commuting vector fields: bound- 
ary behavior of nonnegative solutions, Ann. Sc. Norm. Super. Pisa Cl. Sci. (5) 11 (2012), 437-474.

[21] M. Frentz, K. Nyström, A. PascucCI and S. Polidoro, Optimal regularity in the obstacle problem for Kolmogorov operators related to American Asian options, Math. Ann. (2010), 805-838.

[22] N. GAROFALO, Second order parabolic equations in nonvariational forms: boundary Harnack principle and comparison theorems for nonnegative solutions, Ann. Mat. Pura Appl. 138 (1984), 267-296.

[23] S. Hofmann and J. L. Lewis, "The Dirichlet Problem for Parabolic Operators with Singular drift Terms", Mem. Amer. Math. Soc., Vol. 151, 2001, viii+113.

[24] L. HöRMANDER, Hypoelliptic second order differential equations, Acta Math. 119 (1967), 147-171.

[25] D. S. JERISON and C. E. KENIG, Boundary behavior of harmonic functions in nontangentially accessible domains, Adv. Math. 46 (1982), 80-147.

[26] C. E. KENIG and J. PIPHER, The Dirichlet problem for elliptic equations with drift terms, Publ. Mat. 45 (2001), 199-217.

[27] N. V. KRYLOV and M. V. SAFONOV, A property of the solutions of parabolic equations with measurable coefficients, Izv. Akad. Nauk SSSR Ser. Mat. 44 (1980), 161-175, 239.

[28] E. LANCONELli and S. POLIDORO, On a class of hypoelliptic evolution operators, Rend. Sem. Mat. Univ. Politec. Torino 52 (1994), 29-63. Partial differential equations, II (Turin, 1993).

[29] E. B. LeE and L. MARKUs, "Foundations of Optimal Control Theory", Robert E. Krieger Publishing Co. Inc., Melbourne, FL, second ed., 1986.

[30] M. MANFREDINI, The Dirichlet problem for a class of ultraparabolic equations, Adv. Differential Equations 2 (1997), 831-866.

[31] K. NYSTRÖM, The Dirichlet problem for second order parabolic operators, Indiana Univ. Math. J. 46 (1997), 183-245.

[32] K. NyströM, A. PASCUCCI and S. Polidoro, Regularity near the initial state in the obstacle problem for a class of hypoelliptic ultraparabolic operators, J. Differential Equations 249 (2010), 2044-2060.

[33] M. V. SAFONOV and Y. YUAN, Doubling properties for second order parabolic equations, Ann. of Math. 150 (1999), 313-327.

[34] S. SALSA, Some properties of nonnegative solutions of parabolic differential operators, Ann. Mat. Pura Appl. 128 (1981), 193-206.

\author{
Dipartimento di Matematica \\ Università di Bologna \\ Piazza di Porta S. Donato 5 \\ 40126 Bologna, Italia \\ cinti@dm.unibo.it \\ Department of Mathematics \\ Uppsala University \\ 75106 Uppsala, Sweden \\ kaj.nystrom@math.uu.se \\ Dipartimento di Scienze Fisiche \\ Informatiche e Matematiche \\ Università di Modena e Reggio Emilia \\ via Campi 213/b \\ 41115 Modena, Italia \\ sergio.polidoro@unimore.it
}

